\title{
THE FOURIER EXTENSION OPERATOR ON LARGE SPHERES AND RELATED OSCILLATORY INTEGRALS
}

\author{
JONATHAN BENNETT AND ANDREAS SEEGER
}

\begin{abstract}
We obtain new estimates for a class of oscillatory integral operators with folding canonical relations satisfying a curvature condition. The main lower bounds showing sharpness are proved using Kakeya set constructions. As a special case of the upper bounds we deduce optimal $L^{p}\left(\mathbb{S}^{2}\right) \rightarrow L^{q}\left(R \mathbb{S}^{2}\right)$ estimates for the Fourier extension operator on large spheres in $\mathbb{R}^{3}$, which are uniform in the radius $R$. Two appendices are included, one concerning an application to Lorentz space bounds for averaging operators along curves in $\mathbb{R}^{3}$, and one on bilinear estimates.
\end{abstract}

\section{INTRODUCTION}

For functions $g \in L^{1}\left(\mathbb{S}^{d}\right)$ on the $d$-dimensional unit sphere we define the Fourier extension operator to be the mapping $\mathcal{E}: g \mapsto \widehat{g d \sigma}$ where

$$
\widehat{g d \sigma}(\xi)=\int_{\mathbb{S}^{d}} e^{-i\langle x, \xi\rangle} g(x) d \sigma(x),
$$

$d \sigma$ denotes the rotation invariant measure on $\mathbb{S}^{d}$ induced by Lebesgue measure in $\mathbb{R}^{d+1}$, and $\xi \in \mathbb{R}^{d+1}$. We note that the adjoint of this operator is the Fourier restriction operator $\left.f \mapsto \widehat{f}\right|_{\mathbb{S}^{d}}$, where $\widehat{ }$ denotes the Euclidean Fourier transform in $d+1$ dimensions. A substantial amount of recent work is concerned with weighted inequalities of the general form

$$
\left(\int|\widehat{g d \sigma}|^{q} d \mu\right)^{1 / q} \lesssim\|g\|_{L^{p}\left(\mathbb{S}^{d}\right)}
$$

for certain measures $\mu$ on $\mathbb{R}^{d+1}{ }^{1}$ Perhaps the most notable instance of this is the case of Lebesgue measure, which corresponds to the classical Fourier restriction problem; see for example [20, 36], 39, [8] and [38. In addition to this, the inequalities (1.1) for certain broader classes of measures $\mu$ are known to have applications to a variety of wellknown and largely unsolved problems in partial differential equations, harmonic analysis and geometric measure theory; see [4, [33, 12], 13, 41], 10, 35, 26], 19], 18, and many further references contained in those papers. The content of the current paper is partially motivated by the particular situation where the measures $\mu$ are supported on large spheres in $\mathbb{R}^{d+1}$; this has been studied recently in [2], [3] and [6]. We take $\mu$ to be the rotation invariant measure on $\lambda S^{d}$ induced by Lebesgue measure in $\mathbb{R}^{d+1}$. In particular the case for circles in the plane is well understood; namely the $L^{p}\left(\mathbb{S}^{1}\right) \rightarrow L^{q}\left(\lambda \mathbb{S}^{1}\right)$ operator norm of $\mathcal{E}$ is uniformly bounded in $\lambda$, if, and only if, $q \geq 3$ and $p \geq q /(q-2)$. This follows

Research partially supported by EPSRC Postdoctoral Fellowship GR/S27009/02 (J.B.) and by the National Science Foundation (A.S.).

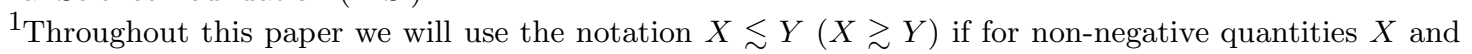
$Y$ there exists a constant $C>0$ such that $X \leq C Y(X \geq C Y)$. The dependence of the implicit constant $C$ on various parameters present will be clarified by the context. 
from a result on more general oscillatory integral operators in 22] for further discussion and an alternative proof of the $L^{3}$ bound see [6]. Here we prove for spheres in $\mathbb{R}^{3}$ :

Theorem 1.1. The inequality

$$
\|\widehat{g d \sigma}\|_{L^{q}\left(\lambda \mathbb{S}^{2}\right)} \leq C\|g\|_{L^{p}\left(\mathbb{S}^{2}\right)}
$$

holds for all $\lambda$, all $g \in L^{p}\left(\mathbb{S}^{2}\right)$ and some $C$, if and only if $q>5 / 2$ and $p \geq 2 q /(2 q-3)$.

After rescaling one sees that uniform $L^{p}\left(\mathbb{S}^{d}\right) \rightarrow L^{q}\left(\lambda \mathbb{S}^{d}\right)$ bounds for $\mathcal{E}$ are equivalent with the $O\left(\lambda^{-d / q}\right)$ bound for the $L^{p}\left(\mathbb{S}^{d}\right) \rightarrow L^{q}\left(\mathbb{S}^{d}\right)$ operator norm of $\mathcal{E}_{\lambda}$, given by

$$
\mathcal{E}_{\lambda} g(\xi)=\widehat{g d \sigma}(\lambda \xi) \text {. }
$$

The operators $\mathcal{E}$ and $\mathcal{E}_{\lambda}$ are closely related to a Radon transform arising in scattering theory, considered by Melrose and Taylor [27. After appropriately parametrizing $\mathbb{S}^{d}$ the operator $\mathcal{E}_{\lambda}$ may be seen as a special case of a much more general class of oscillatory operators acting on functions defined on $\mathbb{R}^{d}$, given by

$$
T_{\lambda} f(x)=\int e^{i \lambda \phi(x, y)} \chi(x, y) f(y) d y .
$$

Here $\phi$ is a smooth real-valued phase function on $\Omega_{L} \times \Omega_{R}$ where $\Omega_{L}$ and $\Omega_{R}$ are open subsets of $\mathbb{R}^{d}$ and $\chi$ is smooth with supp $\chi \subset \Omega_{L} \times \Omega_{R}$. We shall now discuss the assumptions on the phase which are appropriate for the study of $\mathcal{E}_{\lambda}$.

The $L^{2}$ mapping properties of $T_{\lambda}$ are governed by geometrical properties of the canonical relation associated to the phase $\phi$; it is defined to be the (twisted) graph of the gradient map,

$$
\mathcal{C}_{\phi}=\left\{\left(x, \nabla_{x} \phi, y,-\nabla_{y} \phi\right):(x, y) \in \operatorname{supp} \chi\right\} \subset T^{*} \Omega_{L} \times T^{*} \Omega_{R} .
$$

Here we assume that the projections $\pi_{L}$ and $\pi_{R}$ mapping $\mathcal{C}_{\phi}$ to $T^{*} \Omega_{L}$ and $T^{*} \Omega_{R}$, respectively,

$$
\begin{aligned}
& \pi_{L}:(x, y) \mapsto\left(x, \phi_{x}(x, y)\right) \\
& \pi_{R}:(x, y) \mapsto\left(x, \phi_{y}(x, y)\right)
\end{aligned}
$$

are Whitney folds. Analytically the fold condition on $\pi_{L}$ can be expressed by requiring that corank $d \pi_{L} \leq 1$ and when dimension ker $d \pi_{L}=1$ then the Hessian considered as a map from ker $d \pi_{L}$ to coker $d \pi_{L}$ is nonzero; i.e.

$$
0 \neq b \in \operatorname{ker} \phi_{x y}, 0 \neq a \in \text { coker } \phi_{x y}, \quad \Longrightarrow \quad\left\langle b, \nabla_{y}\right\rangle^{2}\left\langle a, \phi_{x}\right\rangle \neq 0 .
$$

An equivalent condition is

$$
\operatorname{det} \phi_{x y}(x, y)=0,0 \neq b \in \operatorname{ker} \phi_{x y}, \quad \Longrightarrow \quad\left\langle b, \nabla_{y}\right\rangle\left(\operatorname{det} \phi_{x y}\right) \neq 0 .
$$

Similarly the corresponding condition on $\pi_{R}$ being a Whitney fold is

$$
\operatorname{det} \phi_{y x}(x, y)=0,0 \neq a \in \operatorname{ker} \phi_{y x}, \quad \Longrightarrow \quad\left\langle a, \nabla_{x}\right\rangle\left(\operatorname{det} \phi_{y x}\right) \neq 0 .
$$

Using the terminology in 27] we say that $\mathcal{C}_{\phi}$ is a folding canonical relation if (1.7) and (1.8) are satisfied. The $L^{2}$ operator norm of $T_{\lambda}$ is $O\left(\lambda^{-d / 2+1 / 6}\right)$ by the work of Melrose-Taylor 27] and Pan-Sogge 31].

Condition (1.7) makes

$$
\mathcal{L}=\left\{(x, y): \operatorname{det} \phi_{x y}=0\right\}
$$

a smooth hypersurface in $\mathbb{R}^{d} \times \mathbb{R}^{d}$; moreover for fixed $x$

$$
\{y:(x, y) \in \mathcal{L}\}
$$


is a smooth hypersurface in $\mathbb{R}^{d}$, and thus the varieties

$$
\mathcal{L}_{x}:=\left\{\xi \in T_{x}^{*} \Omega_{L}: \xi=\phi_{x}(x, y),(x, y) \in \mathcal{L}\right\}
$$

are smooth hypersurfaces in the fibers. Following [21] we assume the following condition (which is based on the Carleson-Sjölin hypothesis, cf. [24], 28]):

\section{Curvature condition:}

For every $x \in \Omega_{L}$, the hypersurface $\mathcal{L}_{x}$ is convex and has nonvanishing curvature.

The convexity and nonvanishing curvature hypotheses mean that the second fundamental form is either positive definite or negative definite everywhere on $\mathcal{L}_{x}$.

Condition (1.12) is not relevant for $L^{2} \rightarrow L^{2}$ bounds, however it is crucial for $L^{p} \rightarrow L^{q}$ bounds in higher dimensions. In one dimension there is no curvature condition and the best possible results are known, namely

$$
\left\|T_{\lambda}\right\|_{L^{p}(\mathbb{R}) \rightarrow L^{q}(\mathbb{R})} \lesssim \lambda^{-1 / q}, \quad q \geq 2 p^{\prime}, q \geq 3,
$$

holds under the assumptions (1.7), (1.8). This was proved in 22. Examples (see (3) show that the sharp bound

$$
\left\|T_{\lambda}\right\|_{L^{p}\left(\mathbb{R}^{d}\right) \rightarrow L^{q}\left(\mathbb{R}^{d}\right)} \lesssim \lambda^{-d / q},
$$

can only hold for $q \geq(d+1) p^{\prime} / d$ and $q \geq(2 d+1) / d$ (here $\left.p^{\prime}=p /(p-1)\right)$. In two and higher dimensions Kakeya type examples exclude the case $q=(2 d+1) / d$. Under assumption (1.12) inequality (1.14) has been established by Greenleaf and one of the authors 21] in the range $q \geq(2 d+2) / d$; actually in [21] the assumption of a folding canonical relation has been replaced with a weaker one-sided assumption involving only the projection $\pi_{L}$. Moreover, in the range $q \geq(2 d+2) / d$ the definiteness assumption on the second fundamental form can be replaced by merely the nondegeneracy assumption (of course this makes no difference when $d=2$ ).

Under the folding relation and curvature assumptions we improve the known range $q \geq 3$ of inequality (1.14) in two dimensions, and get a best possible result.

Theorem 1.2. Suppose that $d=2$ that $\mathcal{C}_{\phi}$ is a folding canonical relation and that the curvature condition (1.12) is satisfied. Then for $\lambda \geq 2$

$$
\left\|T_{\lambda}\right\|_{L^{p}\left(\mathbb{R}^{2}\right) \rightarrow L^{q}\left(\mathbb{R}^{2}\right)} \lesssim \lambda^{-2 / q}, \quad q \geq \frac{3 p^{\prime}}{2}, \quad q>\frac{5}{2} .
$$

Moreover,

$$
\left\|T_{\lambda}\right\|_{L^{q}\left(\mathbb{R}^{2}\right) \rightarrow L^{q}\left(\mathbb{R}^{2}\right)} \lesssim \lambda^{-\frac{2}{3}-\frac{1}{3 q}}(\log \lambda)^{\frac{1}{2}-\frac{1}{q}}, \quad 2 \leq q<5 / 2,
$$

and

$$
\left\|T_{\lambda}\right\|_{L^{5 / 2,1}\left(\mathbb{R}^{2}\right) \rightarrow L^{5 / 2, \infty}\left(\mathbb{R}^{2}\right)} \lesssim \lambda^{-4 / 5}(\log \lambda)^{1 / 10} .
$$

The estimates are sharp in the following sense: If there is a point $P \in \mathcal{L}$ so that $\chi(P) \neq 0$ then there is a positive constant $c>0$ depending on $\chi$ and $\lambda_{0}>1$ so that for all $\lambda \geq \lambda_{0}$

$$
\left\|T_{\lambda}\right\|_{L^{p, 1}\left(\mathbb{R}^{2}\right) \rightarrow L^{q, \infty}\left(\mathbb{R}^{2}\right)} \geq c \max \left\{\lambda^{-\frac{2}{q}}, \lambda^{-\frac{2}{3 p^{\prime}}-\frac{1}{q}}, \lambda^{-\frac{2}{3}-\frac{1}{3 q}}(\log \lambda)^{\frac{1}{2}-\frac{1}{q}}\right\} .
$$

It would be interesting to know whether the restricted weak type estimate (1.17) could be replaced by an $L^{5 / 2} \rightarrow L^{5 / 2}$ estimate with the same bounds; this remains open.

The assumptions of Theorem 1.2 are satisfied for the operator $\mathcal{E}_{\lambda}$ in the Fourier extension problem on spheres, so that Theorem 1.1] is a direct consequence of Theorem 1.2 (see \$2). 
Indeed the spheres on both sides of inequality (1.2) may be replaced by compact pieces of two surfaces in $\mathbb{R}^{3}$ with nonvanishing Gaussian curvature.

Structure of the paper. In $\$ 2$ we discuss some preparatory changes of variables which are useful in the proof of both the necessary and sufficient conditions, and briefly discuss the validity of our assumptions for the phases in the Fourier extension problem. In $\$ 3$ we prove the sharpness of Theorem 1.2 the main part of this section is concerned with a Kakeya type example. In $\$ 4$ we give the basic decompositions of the operator in terms of the size of det $\phi_{x y}^{\prime \prime}$ and state the main estimates for these pieces. In $\$ 5$ we discuss easy proofs of the required bounds in certain model cases and raise some open questions. The more technical proof of the main estimates in the general case is given in $\$ 6$ and $\$ 8$. The paper has two appendices. In the first one, 99 , we consider the convolution with measures on some curves in $\mathbb{R}^{3}$; we use a variant of our estimates to give a Lorentz-space improvement of Oberlin's endpoint estimates 30. In the second appendix, 10 , we revisit the bilinear estimates from 2] and give a straightforward proof based on the geometric properties of the canonical relation.

\section{Acknowledgements:}

J.B. would like to thank Juan Antonio Barceló, Tony Carbery, Fernando Soria and Ana Vargas for their on-going collaborative work on the subject of general weighted $L^{2}$ norm inequalities for the Fourier extension operator. The $L^{p}\left(\mathbb{S}^{d}\right) \rightarrow L^{q}\left(R \mathbb{S}^{d}\right)$ Fourier extension problems addressed in this paper arose naturally in this work. Thanks are also due to Tony Carbery for his involvement in the early stages of this project.

A.S. would like to thank Allan Greenleaf for numerous conversations on oscillatory integral operators, many of them related to this project, in the course of their long term collaboration.

\section{Preparation of the phase FunCtion}

It is advantageous to suitably prepare the phase function by possibly changing variables in $x$ and in $y$. These changes of variables affect the estimates only by constants. We have to observe that our hypotheses are invariant under these changes of variables. This is standard for the conditions (1.7) and (1.8). Concerning the curvature condition a change of variables in $x$ induces a linear change in the fiber $(\xi-)$ variables and thus leaves the curvature condition invariant. We now examine the independence of parametrization and invariance under change of the $y$-variable, of the curvature condition. We shall consider the situation in $d$ dimensions.

If $x$ is fixed and $z \mapsto G(x, z)$ is a regular parametrization of $\{y:(x, y) \in \mathcal{L}\}$ (with parameter $z \in \mathbb{R}^{d-1}$ ) then vectors in coker $\phi_{x y}$ are normal to the hypersurface $\mathcal{L}_{x}$ in the fiber above $x$ and the curvature condition is just saying that for $v \in$ coker $\phi_{x y}$ the Hessian of the map

$$
z \mapsto\left\langle v, \nabla_{x} \phi(x, G(x, z))\right\rangle
$$

is either positive definite or negative definite; this Hessian equals

$$
\frac{\partial G}{\partial z}^{T}\left\langle v, \nabla_{x} \phi\right\rangle_{y y}^{\prime \prime} \frac{\partial G}{\partial z}+O\left(\left\langle v, \phi_{x y}\right\rangle\right)
$$

at $y=G(x, z)$ and the last term drops out since $v \in$ coker $\phi_{x y}(x, G(x, z))$. From this the invariance easily follows. 
We now prepare our phase function to have an approximate normal form at a point $P=\left(x^{o}, y^{o}\right)$, and we may assume that $P \in \mathcal{L}$. (i.e. to have certain derivatives vanish at $P)$. Let us assume that the phase function

$$
(x, y) \mapsto \psi(x, y)
$$

has a canonical relation $\mathcal{C}_{\psi}$ satisfying (1.7), (1.8), and (1.12). We shall find diffeomorphisms $G_{L}$ and $G_{R}$, mapping neighborhoods of the origins of $\mathbb{R}_{L}^{d}, \mathbb{R}_{R}^{d}$, to neighborhoods of $x^{o}, y^{o}$ respectively, so that at the origin $O=\left(O_{L}, O_{R}\right)$ the phase

$$
\phi(x, y)=\psi\left(G_{L}(x), G_{R}(y)\right)
$$

satisfies the conditions

$$
\operatorname{det} \phi_{x^{\prime} y^{\prime}}(O) \neq 0
$$

and

$$
\begin{aligned}
& \phi_{x y_{d}}(O)=0 \\
& \phi_{x_{d} y}(O)=0 ;
\end{aligned}
$$

moreover

$$
\begin{aligned}
& \phi_{x_{d} y_{d} y_{d}}(O) \neq 0, \\
& \phi_{x_{d} x_{d} y_{d}}(O) \neq 0, \\
& \phi_{x_{d} y_{d} y^{\prime}}(O)=0, \\
& \phi_{x_{d} y_{d} x^{\prime}}(O)=0,
\end{aligned}
$$

and also

$$
\phi_{x^{\prime} y^{\prime} x_{d}}(O)=0 .
$$

To accomplish this, let $a$ and $b$ be unit vectors in $\mathbb{R}_{L}^{d}$ and $\mathbb{R}_{R}^{d}$ respectively, so that at $P$ we have $\psi_{x y} b=0, a^{T} \psi_{x y}=0$ (recall that we assume that at $P$ the kernel and cokernel of $\psi_{x y}$ are one dimensional). Now choose rotations $\rho_{L}$ of $\mathbb{R}_{L}^{d}$ and $\rho_{R}$ of $\mathbb{R}_{R}^{d}$ so that $\rho_{L}^{-1} b=e_{d}$, $\rho_{R}^{-1} a=e_{d}$. Then

$$
\phi^{[1]}(x, y)=\psi\left(x^{o}+\rho_{L}(x), y^{o}+\rho_{R}(y)\right)
$$

satisfies $\operatorname{det} \phi_{x^{\prime} y^{\prime}}^{[1]}(O) \neq 0, \phi_{x^{\prime} y_{d}}^{[1]}(O)=0$ and $\phi_{x_{d} y^{\prime}}^{[1]}(O)=0$. By the formula

$$
\operatorname{det} \phi_{x y}=\operatorname{det}\left(\phi_{x^{\prime} y^{\prime}}\right)\left(\phi_{x_{d} y_{d}}-\phi_{x_{d} y^{\prime}} \phi_{x^{\prime} y^{\prime}}^{-1} \phi_{x^{\prime} y_{d}}\right)
$$

(applied to $\left.\phi^{[1]}\right)$ we also have $\phi_{x_{d} y_{d}}^{[1]}(O)=0$ and see that $\phi^{[1]}$ satisfies (2.2), (2.3) and (2.4). Notice that from the fold assumptions (1.7) and (1.8) we also have $\phi_{x_{d} y_{d} y_{d}}^{[1]}(O) \neq 0$, and $\phi_{x_{d} x_{d} y_{d}}^{[1]}(O) \neq 0$.

We now consider the phase-function

$$
\phi^{[2]}(x, y)=\phi^{[1]}\left(\sigma_{L}(x), \sigma_{R}(y)\right)
$$

for suitable shears in $\mathbb{R}_{L}^{d}$ and $\mathbb{R}_{R}^{d}$, of the form

$$
\sigma_{L}(x)=\left(x^{\prime}, x_{d}-\sum_{i=1}^{d-1} \alpha_{i} x_{i}\right), \quad \sigma_{R}(y)=\left(y^{\prime}, y_{d}-\sum_{j=1}^{d-1} \beta_{j} y_{j}\right)
$$


Note that $\phi^{[2]}$ still satisfies (2.2), (2.3) and (2.4), and also (2.5) and (2.6), independently of the choice of $\alpha$ and $\beta$. Now if we choose

$$
\alpha_{i}=-\frac{\phi_{x_{d} y_{d} x_{i}}^{[1]}(O)}{\phi_{x_{d} y_{d} x_{d}}^{[1]}(O)}, \quad \beta_{j}=-\frac{\phi_{x_{d} y_{d} y_{j}}^{[1]}(O)}{\phi_{x_{d} y_{d} y_{d}}^{[1]}(O)},
$$

then conditions (2.7), (2.8) are satisfied for $\phi^{[2]}$ as well.

Now set

$$
\phi(x, y)=\phi^{[2]}\left(\left(x^{\prime}+x_{d} B x^{\prime}, x_{d}\right), y\right)
$$

where $B=-\left[\phi_{x^{\prime} y^{\prime}}^{[2]}(O)\right]^{-1} \phi_{y^{\prime} x^{\prime} x_{d}}^{[2]}(O)$. Then (2.11) holds with $G_{R}(y)=y^{0}+\rho_{R}\left(\sigma_{R}(y)\right)$ and $G_{L}(x)=x^{0}+\rho_{L}\left(\sigma_{L}(\nu(x))\right)$, where $\nu(x)=\left(x^{\prime}+x_{d} B x^{\prime}, x_{d}\right)$. The phase $\phi$ satisfies (2.9) and conditions (2.2) - (2.8) continue to hold.

Finally, by replacing the phase $\phi(x, y)$ with $\phi(x, y)-\phi\left(x^{o}, y\right)$ we may assume that

$$
\partial_{y}^{\alpha} \phi(O)=0
$$

for all multiindices $\alpha$.

We now examine the curvature condition (1.12) at $O$. By condition (2.5) we can solve near $O$

$$
\operatorname{det} \phi_{x y}=0 \quad \Longleftrightarrow \quad y_{d}=g\left(x, y^{\prime}\right)
$$

with $g\left(O_{L}, O_{R}^{\prime}\right)=0$. Implicit differentiation and condition (2.7) implies that

$$
\nabla_{y^{\prime}} g\left(O_{L}, O_{R}^{\prime}\right)=0 .
$$

Thus our curvature condition at $O$ reads

$$
\nabla_{y^{\prime} y^{\prime}}^{2}\left(\left.\phi_{x_{d}}\left(O_{L}, y^{\prime}, g\left(O_{L}, y^{\prime}\right)\right)\right|_{y^{\prime}=O_{R}^{\prime}}\right. \text { is positive or negative definite, }
$$

which by (2.7) and (2.13) reduces to the definiteness assumption on the Hessian of $\phi_{x_{d}}$, namely,

$$
\nabla_{y^{\prime} y^{\prime}}^{2} \phi_{x_{d}}(P) \text { is positive or negative definite. }
$$

On the phase functions in the Fourier extension problem. We briefly discuss here how the extension operator $\mathcal{E}_{\lambda}$ in (1.3) of the introduction belongs to our general family of oscillatory integral operators $T_{\lambda}$ satisfying (1.7), (1.8) and (1.12).

Let $S$ be a patch of a smooth convex hypersurface of $\mathbb{R}^{d+1}$, with nonvanishing Gaussian curvature (in particular $S$ may be part of $\mathbb{S}^{d}$ as in (1.3)). Let $y \mapsto \Gamma(y)$ be a parametrization of $S$ (where the parameter $y$ is chosen from an open subset of $\mathbb{R}^{d}$ ). Let $\Sigma$ be a smooth hypersurface of $\mathbb{R}^{d+1}$, parametrized by $x \mapsto \Xi(x)$, where $x$ belongs to an open set of $\mathbb{R}^{d}$. Then the operator $\mathcal{E}_{\lambda}$ in (1.3) may now be written as an oscillatory integral operator with phase function

$$
\phi(x, y)=\langle\Xi(x), \Gamma(y)\rangle .
$$

Clearly $\phi_{x y}=\Xi^{\prime}(x)^{T} \Gamma^{\prime}(y)$ is of rank $\geq d-1$ and $\mathcal{L}$ consists of those $(x, y)$ for which the normal line for $S$ at $\Gamma(y)$ is parallel to the tangent space for $\Sigma$ at $\Xi(x)$ (or, equivalently, the normal line for $\Sigma$ at $\Xi(x)$ is parallel to the tangent space for $S$ at $\Gamma(y)$ ).

The assumption that the second fundamental form of $S$ is definite implies that the fold condition for $\pi_{L}$, (1.7), is satisfied. Indeed if $(x, y) \in \mathcal{L}$ and if $a, b$ are nonzero vectors in $\mathbb{R}^{d}$ so that $a^{T} \Xi^{\prime}(x)^{T} \Gamma^{\prime}(y)=0$ and $\Xi^{\prime}(x)^{T} \Gamma^{\prime}(y) b=0$ then the fold condition in the form (1.6) is saying that

$$
\left\langle b, \nabla_{y}\right\rangle a^{T} \Xi^{\prime}(x)^{T} \Gamma^{\prime}(y) b \neq 0
$$


and this is implied by the definiteness of the fundamental form of $S$ since $\Xi^{\prime}(x) a$ is a nonzero vector perpendicular to the tangent space of $S$ at $\Gamma(y)$.

For the curvature condition (1.12) we fix $x$ and solve $\operatorname{det} \phi_{x y}=0$ by $y=G(x, z)$ so that $\mathcal{L}_{x}$ is parametrized by $z \mapsto \Xi^{\prime}(x)^{T} \Gamma(G(x, z))$. We need to verify that the second fundamental form of $\mathcal{L}_{x}$ is definite, i.e. that

$$
\nabla_{z z}^{2}\left\langle a, \Xi^{\prime}(x)^{T} \Gamma(G(x, z))\right\rangle
$$

is definite if $\left\langle a, \Xi^{\prime}(x)^{T} \Gamma^{\prime}(G(x, z))\right\rangle=0$. However under this last condition the second fundamental form becomes

$$
\frac{\partial G}{\partial z}^{T} \nabla_{y y}^{2}\left\langle\Xi^{\prime}(x) a, \Gamma(y)\right\rangle \frac{\partial G}{\partial z} \text { at } y=G(x, z) .
$$

Again as $\Xi^{\prime}(x) a$ is normal to $S$ at $\Gamma(y)$ we see by the definiteness assumption on the second fundamental form and by $\operatorname{rank} \frac{\partial G}{\partial z}=d-1$, that the last displayed formula gives a definite $(d-1) \times(d-1)$ matrix. Thus the curvature condition is verified.

Finally, if in addition we also assume that $\Sigma$ is a convex hypersurface with nonvanishing curvature then we see by symmetry that the fold condition for $\pi_{R}$, (1.8), is satisfied as well (see also [15] for a discussion of the structure of $\pi_{R}$ in the more general situation where $\Sigma$ is convex and of finite line type).

\section{LOWER BOUNDS}

We now establish lower bounds for the operator norms of $T_{\lambda}$ showing in particular the sharpness of Theorem 1.2. We work in $d$ dimensions and assume that the fold and curvature conditions (1.7), (1.8) and (1.12) hold, and in addition we make the (necessary) assumption that there is a point $\left(x^{o}, y^{o}\right) \in \mathcal{L}$ for which

$$
\chi\left(x^{o}, y^{o}\right) \neq 0 .
$$

By the reductions described in $\oint 2$ we may assume that $\left(x^{o}, y^{o}\right)=O$, that (2.2 2.9) hold, and, in dimension $d \geq 2$, that (2.15) holds.

We are interested in the range of exponents $(p, q)$ for which

$$
\left\|T_{\lambda}\right\|_{L^{p, 1} \rightarrow L^{q, \infty}} \lesssim \lambda^{-d / q}
$$

holds. It is easy to see that the decay rate in (3.2) is sharp (for any $C^{1}$ phase function). Since the operator is local we have

$$
\left\|T_{\lambda}\right\|_{L^{\infty} \rightarrow L^{q, \infty}} \lesssim\left\|T_{\lambda}\right\|_{L^{p, 1} \rightarrow L^{q, \infty}}
$$

and therefore it suffices to prove lower bounds for the weak type $(\infty, q)$ operator norm. Without loss of generality $\operatorname{Re}(\chi(x, y))>c>0$ for $|x| \leq \varepsilon,|y| \leq \varepsilon$. Let $\lambda \gg \varepsilon^{-1}$, and define $f(y)=e^{-i \lambda \phi(0, y)}$ for $|y| \leq \varepsilon$ and $f(y)=0$ elsewhere. Then $\left|T_{\lambda} f(x)\right| \geq c>0$ for $|x| \leq c_{0} \varepsilon \lambda^{-1}$ and thus

$$
\left\|T_{\lambda}\right\|_{L^{\infty} \rightarrow L^{q, \infty}} \geq c^{\prime} \lambda^{-d / q} .
$$

The following simple lemma shows that the condition $q \leq(d+1) p^{\prime} / d$ is necessary for (3.2) to hold. Note that (3.4) and (3.5) yield the first two lower bounds stated in (1.18).

Lemma 3.1. There is $c>0$ so that

$$
\left\|T_{\lambda}\right\|_{L^{p, 1} \rightarrow L^{q, \infty}} \geq c \lambda^{d /(3 p)-d / 3-(2 d-1) /(3 q)} .
$$


Proof. Let $f_{0}$ be the characteristic function of the ball $\left\{y:|y| \leq \varepsilon \lambda^{-1 / 3}\right\}$, and define

$$
f(y)=f_{0}(y) \exp \left(-i \lambda\left(\left\langle y, \phi_{y}(O)\right\rangle+\frac{1}{2}\left\langle y, \phi_{y y}(O) y\right\rangle\right)\right)
$$

so that $\|f\|_{L^{p, 1}} \approx \lambda^{-d /(3 p)}$. By considering the Taylor expansion of $\phi(x, y)-\phi(x, 0)$ we observe that

$$
\left|\phi(x, y)-\phi(x, 0)-\left\langle y, \phi_{y}(O)\right\rangle-\frac{1}{2}\left\langle y, \phi_{y y}(O) y\right\rangle\right| \leq C \varepsilon \lambda^{-1}
$$

whenever $|y| \leq \varepsilon \lambda^{-1 / 3},|x| \leq \varepsilon \lambda^{-1 / 3}$ and $\left|\left\langle x, \phi_{x y}(O) y\right\rangle\right| \leq \lambda^{-1}$. On multiplying $T_{\lambda} f(x)$ by the unimodular factor $e^{-i \lambda \phi(x, 0)}$, we find that if $x$ is such that these conditions hold uniformly in $|y| \leq \varepsilon \lambda^{-1 / 3}$, then

$$
\left|T_{\lambda} f(x)\right| \geq c \lambda^{-d / 3}
$$

if $\varepsilon$ is sufficiently small. By the assumptions (2.3) and (2.4) we see that $\left|\left\langle x, \phi_{x y}(O) y\right\rangle\right| \leq \lambda^{-1}$ holds for all $|y| \leq \varepsilon \lambda^{-1 / 3}$ whenever $\left|x^{\prime}\right| \leq \varepsilon^{\prime} \lambda^{-2 / 3}$ and $\left|x_{d}\right| \leq \varepsilon^{\prime} \lambda^{-1 / 3}$. Thus $\left\|T_{\lambda} f\right\|_{L^{q, \infty}} \gtrsim$ $\lambda^{-d / 3-(2 d-1) /(3 q)}$, and the assertion follows.

We shall now show by a randomization argument that for $d \geq 2$ the inequality (3.2) can only hold for $q>(2 d+1) / d$, and also establish the sharpness of (1.16), (1.17). The approach is inspired by the result of Beckner, Carbery, Semmes and Soria [5] on the failure of restricted weak type endpoint bounds for the classical Fourier extension operator ( $c f$. also Tao's generalization [37] to oscillatory integral operators). We use a rescaled version of the Kakeya construction in Keich [25]. Let $\delta \ll 1, \delta<\alpha<1 / 10$ and suppose that for every $n^{\prime} \in \mathbb{Z}^{d-1}$ with $\left|n^{\prime} \delta\right| \leq \alpha$ we are given a $r \delta \times \cdots \times r \delta \times r$ rectangle $P_{n}$ passing through the hyperplane $x_{d}=0$ so that the long edges are parallel to $\left(n^{\prime} \delta, 1\right)$. Then there are vectors $v_{n} \in \mathbb{R}^{d-1} \times\{0\},\left|v_{n}\right| \leq|\alpha|$, so that the union of translated rectangles $v_{n}+P_{n}$ satisfies

$$
\left|\bigcup_{n} v_{n}+P_{n}\right| \leq C(\log (\alpha / \delta))^{-1} \sum_{n}\left|P_{n}\right| \text {. }
$$

We shall apply this fact after possible changes of variables, with $r=\delta=\lambda^{-1 / 3}, \alpha=\epsilon \lambda^{-1 / 6}$, and large $\lambda$, then $\log (\alpha / \delta) \approx \log \lambda$.

Proposition 3.2. Suppose $d \geq 2$ and $q>2$, then there is $c>0$ and $\lambda_{0}>0$ so that for all $\lambda>\lambda_{0}$

$$
\left\|T_{\lambda}\right\|_{L^{\infty} \rightarrow L^{q, \infty}} \geq c \lambda^{-d / 3-(d-1) /(3 q)}(\log \lambda)^{1 / 2-1 / q} .
$$

Proof. Assume without loss of generality $\operatorname{Re}(\chi(x, y)) \geq 1$ whenever $\left|x_{i}\right| \leq c_{0},\left|y_{j}\right| \leq c_{0}$ for some constant $c_{0}>0$. Assume $\lambda \gg c_{0}^{-1}$. We let $\mathfrak{Q}$ be the family of all cubes of $\mathbb{R}^{d}$ of sidelength $\lambda^{-1 / 6}$, of the form $\prod_{i=1}^{d}\left[n_{i} \lambda^{-1 / 6},\left(n_{i}+1\right) \lambda^{-1 / 6}\right)$ where $n=\left(n_{1}, \ldots, n_{d}\right) \in \mathbb{R}^{d}$ and $\left|n_{i}\right| \lambda^{-1 / 6} \leq c_{0} / 2$. For $Q \in \mathfrak{Q}$ let $x_{Q}$ be the center of $Q$. Let $y_{Q}=\left(x_{Q}^{\prime}, g\left(x_{Q}, x_{Q}^{\prime}\right)\right)$ where $g$ is given by (2.12), and let $B(Q)$ be the ball of radius $\varepsilon_{1} \lambda^{-1 / 6}$ centered at $y_{Q}$. In view of (2.5) and (2.6) we have $g_{x_{d}} \neq 0$ near the origin and by choosing $c_{0}$ sufficiently small we may assume that $y \mapsto\left(y^{\prime}, g\left(y, y^{\prime}\right)\right)$ is a diffeomorphism near the origin. Consequently, if $\varepsilon_{1}$ is sufficiently small, the balls $\{B(Q): Q \in \mathfrak{Q}\}$ form a disjoint family.

On each cube $Q$, and each ball $B(Q)$ we shall now change variables as in $\S 2$ Namely, for each $Q \in \mathfrak{Q}$ there is a diffeomorphism $\mathfrak{v}_{Q}$ mapping a neighborhood $\mathcal{U}_{L, Q}$ of the origin $O_{L}$ to an open set $\mathcal{V}_{Q}$ containing $Q$ and a diffeomorphism $\mathfrak{w}_{Q}$ mapping a neighborhood $\mathcal{U}_{R, Q}$ of the origin $O_{R}$ to a neighborhood $\mathcal{W}_{Q}$ of $y_{Q}$ containing $B(Q)$, so that the phase function

$$
\psi^{Q}(x, y)=\phi\left(\mathfrak{v}_{Q}(x), \mathfrak{w}_{Q}(y)\right)
$$


satisfies conditions (2.2 [2.9), and also (2.15) holds for $\psi^{Q}$. The bounds for the derivatives of $\psi^{Q}$ are uniform in $Q$, as are the implicit lower bounds in (2.2), (2.5), (2.6), (2.15) for those functions. We can find a positive $\varepsilon_{2} \ll \varepsilon_{1}$ so that for every $Q$ the sets $\mathcal{U}_{L, Q}$ and $\mathcal{U}_{R, Q}$ contain the cubes of sidelength $\varepsilon_{2}$ centered at the origins $O_{L}$ and $O_{R}$, respectively. Moreover there is a positive $\varepsilon_{3} \leq \varepsilon_{2}$, so that if $Q_{o}$ denotes the cube of sidelength $\varepsilon_{3} \lambda^{-1 / 6}$ centered at $O_{L}$ then $\mathfrak{v}_{Q}\left(Q_{o}\right) \subset \bar{Q}$, for every $Q \in \mathfrak{Q}$. We let $\mathcal{Z}=\left\{n \in \mathbb{Z}^{d}:|n| \leq 10^{-1} \varepsilon_{3} \lambda^{1 / 6}\right\}$.

We decompose this cube $Q_{o}$ into plates at height $\lambda^{-1 / 3} n_{d}$, with $\left|n_{d}\right| \leq \varepsilon_{3} 10^{-1} \lambda^{1 / 6}$. Let $\Pi_{n^{\prime}}$ be the orthogonal projection to the hyperplane orthogonal to $\left(n^{\prime} \lambda^{-1 / 3}, 1\right)$. We now apply the above mentioned construction by Keich (with angular parameter $\alpha \leq \varepsilon_{4} \lambda^{-1 / 6}$, cf. (3.6) $)$. Then for each $n_{d}$ we find a family of $\lambda^{-2 / 3} \times \cdots \times \lambda^{-2 / 3} \times \lambda^{-1 / 3}$ rectangles $\widetilde{R}_{n}=\widetilde{R}_{n^{\prime}, n_{d}}$ so that $\widetilde{R}_{n}$ contains the set

$$
R_{n}=\left\{x:\left|x_{d}-n_{d} \lambda^{-1 / 3}\right| \leq \lambda^{-1 / 3} \varepsilon_{4},\left|\Pi_{n^{\prime}}(x-a(n))\right| \leq \varepsilon_{4} \lambda^{-2 / 3}\right\}
$$

where

$$
a(n)=\left(a^{\prime}(n), a_{d}(n)\right) \in \mathbb{R}^{d-1} \times\left\{\lambda^{-1 / 3} n_{d}\right\}, \text { with }\left|a^{\prime}(n)\right| \leq \varepsilon_{3} \lambda^{-1 / 6},
$$

and, for the measure of

$$
E\left(n_{d}\right)=\bigcup_{n^{\prime}} R_{n^{\prime}, n_{d}}
$$

there is the Besicovich type estimate

$$
\left|E\left(n_{d}\right)\right| \leq C \frac{\lambda^{(d-1) / 6} \lambda^{-(2 d-1) / 3}}{\log \lambda}
$$

uniformly in $n_{d}$. Observe that for $n \in \mathbb{Z}^{d}$ the rectangle $\widetilde{R}_{n}$ lies in the plate at height $n^{\prime} \lambda^{-1 / 3}$, contains the point $a(n) \in \mathbb{R}^{d}$ and has long sides in the direction $\left(n^{\prime} \lambda^{-1 / 3}, 1\right)$.

Sublemma 3.3. If $\varepsilon \ll \varepsilon_{4}$ is sufficiently small then there is $c(\varepsilon)>0$ so that the following holds for $\lambda \geq \varepsilon^{-1}$. For each $Q \in \mathfrak{Q}$ there is a disjoint family of balls $B_{n, Q}, n \in \mathcal{Z}$, each of radius $\varepsilon \lambda^{-1 / 3}$ and contained in $\mathfrak{w}_{Q}^{-1}(B(Q))$, and for each $(Q, n) \in \mathfrak{Q} \times \mathcal{Z}$ there is a smooth function $H_{n, Q}$ defined on $B_{n, Q}$ so that with

$$
f_{n, Q}(y)=\chi_{B_{n, Q}}\left(\mathfrak{w}_{Q}^{-1}(y)\right) e^{-i \lambda H_{n, Q}\left(\mathfrak{w}_{Q}^{-1}(y)\right)}
$$

we have

$$
\left|T_{\lambda} f_{n, Q}(x)\right| \geq c(\varepsilon) \lambda^{-d / 3}, \quad \text { if } x \in \mathcal{R}_{n, Q}:=\mathfrak{v}_{Q}\left(R_{n}\right)
$$

We postpone the proof of the sublemma and continue with the proof of the proposition. We show

$$
\left\|\sum_{n, Q} \chi_{\mathcal{R}_{n, Q}}\right\|_{L^{q / 2, \infty}\left(\mathbb{R}^{d}\right)} \lesssim \lambda^{2 d / 3-d /(3 p)}\left\|T_{\lambda}\right\|_{L^{\infty} \rightarrow L^{q, \infty}}^{2} .
$$

To see (3.11) we follow the argument in [5]. We denote by $\left\{r_{k}\right\}$ the system of Rademacher functions. Choose an injective function $(n, Q) \mapsto k(n, Q)$ with values in the positive integers. By Khinchine's inequality

$$
\left(\sum_{n, Q}\left|T_{\lambda} f_{n, Q}(x)\right|^{2}\right)^{1 / 2} \lesssim \int_{0}^{1}\left|\sum_{n, Q} r_{k(n, Q)}(t) T_{\lambda} f_{n, Q}(x)\right| d t
$$


uniformly in $x, \lambda$. Now by the sublemma $\chi_{\mathcal{R}_{n, Q}} \lesssim \lambda^{2 d / 3}\left|T_{\lambda} f_{n, Q}(x)\right|^{2}$, and hence

$$
\begin{aligned}
& \left\|\sum_{n, Q} \chi_{\mathcal{R}_{n, Q}}\right\|_{L^{q / 2, \infty}\left(\mathbb{R}^{d}\right)} \lesssim \lambda^{2 d / 3}\left\|\sum_{n, Q}\left|T_{\lambda} f_{n, Q}\right|^{2}\right\|_{L^{q / 2, \infty}} \\
& =\lambda^{2 d / 3}\left\|\left(\sum_{n, Q}\left|T_{\lambda} f_{n, Q}\right|^{2}\right)^{1 / 2}\right\|_{L^{q, \infty}}^{2} \lesssim \lambda^{2 d / 3}\left\|\int_{0}^{1}\left|\sum_{n, Q} r_{k(n, Q)}(t) T_{\lambda} f_{n, Q}\right| d t\right\|_{L^{q, \infty}}^{2}
\end{aligned}
$$

and the square root of the right hand side is further estimated by a constant times

$$
\begin{aligned}
\lambda^{d / 3} \| \int_{0}^{1} \mid T_{\lambda} & {\left[\sum_{n} r_{k(n, Q)}(t) f_{n, Q}\right] \mid d t\left\|_{L^{q, \infty}} \leq \lambda^{d / 3} \int_{0}^{1}\right\| T_{\lambda}\left[\sum_{n, Q} r_{k(n, Q)}(t) f_{n, Q}\right] \|_{L^{q, \infty}} d t } \\
& \leq \lambda^{d / 3}\left\|T_{\lambda}\right\|_{L^{\infty} \rightarrow L^{q, \infty}} \int_{0}^{1}\left\|\sum_{n, Q} r_{k(n, Q)}(t) f_{n, Q}\right\|_{L^{\infty}} d t \\
& \lesssim \lambda^{d / 3}\left\|T_{\lambda}\right\|_{L^{\infty} \rightarrow L^{q, \infty}}\left\|\sum_{n, Q}\left|f_{n, Q}\right|\right\|_{L^{\infty}} \\
& \lesssim \lambda^{d / 3}\left\|T_{\lambda}\right\|_{L^{\infty} \rightarrow L^{q, \infty}} .
\end{aligned}
$$

For the last inequality we have used the disjointness of the supports of $f_{n, Q}$ which follows from the disjointness of the balls $B(Q)$, and for each fixed $Q$ from the disjointness of the $B_{n, Q}, n \in \mathcal{Z}$.

Next observe that $\left|\mathcal{R}_{n, Q}\right| \approx\left|R_{n}\right| \approx \lambda^{-2(d-1) / 3-1 / 3}$ and that $\operatorname{card}(\mathcal{Z}) \approx \lambda^{d / 6}, \operatorname{card}(\mathfrak{Q}) \approx$ $\lambda^{d / 6}$ and therefore

$$
\lambda^{-(d-1) / 3} \approx\left\|\sum_{Q \in \mathfrak{Q}} \sum_{n \in \mathcal{Z}} \chi_{\mathcal{R}_{n, Q}}\right\|_{1}
$$

Hence, by the duality of $L^{(q / 2)^{\prime}, 1}$ and $L^{q / 2, \infty}$ and (3.9),

$$
\begin{aligned}
\lambda^{-(d-1) / 3} & \lesssim\left|\bigcup_{Q \in \mathfrak{Q}} \bigcup_{\left(n^{\prime}, n_{d}\right) \in \mathcal{Z}} \mathfrak{v}_{Q}\left(R_{n}\right)\right|^{1-2 / q}\left\|\sum_{Q \in \mathfrak{Q}} \sum_{n \in \mathcal{Z}} \chi_{\mathcal{R}_{n, Q}}\right\|_{L^{q / 2, \infty}} \\
& \lesssim\left[\sum_{Q \in \mathfrak{Q}\left|n_{d}\right| \lesssim \lambda^{1 / 6}}\left|E\left(n_{d}\right)\right|\right]^{1-2 / q} \lambda^{2 d / 3}\left\|T_{\lambda}\right\|_{L^{\infty} \rightarrow L^{q, \infty}} \\
& \lesssim\left(\lambda^{(d+1) / 6} \frac{\lambda^{(d-1) / 6} \lambda^{-(2 d-1) / 3}}{\log \lambda}\right)^{1-2 / q} \lambda^{2 d / 3}\left\|T_{\lambda}\right\|_{L^{\infty} \rightarrow L^{q, \infty}}^{2}
\end{aligned}
$$

which implies

$$
\left\|T_{\lambda}\right\|_{L^{\infty} \rightarrow L^{q, \infty}} \geq c \lambda^{-d / 3-(d-1) /(3 q)}(\log \lambda)^{1 / 2-1 / q}
$$

and thus the assertion.

Proof of Sublemma 3.3. We fix $Q$; our estimates will be uniform in $Q$ and we will generally suppress indices indicating the dependence of the terms on $Q$. For $f$ defined near $O_{R}$ (in particular in $B(Q))$ and for $x \in Q$ we set

$$
\mathcal{T}_{\lambda} f(x)=T_{\lambda}\left[f \circ \mathfrak{w}_{Q}^{-1}\right]\left(\mathfrak{v}_{Q}(x)\right) .
$$

Then

$$
\mathcal{T}_{\lambda} f(x)=\int e^{i \lambda \psi(x, y)} \chi_{1}(x, y) f(y) d y
$$


where $\chi_{1}(x, y)=\chi\left(\mathfrak{v}_{Q}(x), \mathfrak{w}_{Q}(y)\right)\left|\operatorname{det} \mathfrak{w}_{Q}^{\prime}(y)\right|$, and the phase

$$
\psi(x, y) \equiv \psi^{Q}(x, y)=\phi\left(\mathfrak{v}_{Q}(x), \mathfrak{w}_{Q}(y)\right)
$$

satisfies conditions (2.2 2.9). We also note that $\operatorname{det} \psi(x, y)=0$ when $y_{d}=\mathfrak{g}\left(x, y^{\prime}\right)$ and $\mathfrak{g}$ satisfies (2.13) and (2.14) (with $\phi$ replaced by $\psi$ ).

We shall now identify balls $B_{n}$ so that for suitable $f_{n}$ supported on $B_{n}$ the function $\mathcal{T}_{\lambda} f_{n}$ is bounded below by $c \lambda^{-d / 3}$ on $R_{n}$. To achieve this we argue very much as in the proof of Lemma 3.1 and analyze the Taylor expansion of the phase function $\psi(x, y)-\psi(x, b)$ about $x=a$, for suitable $a, b$. Let

$$
H(a, b, y)=\psi_{y}(a, b)(y-b)+\frac{1}{2}(y-b)^{t} \psi_{y y}(a, b)(y-b) .
$$

Then

and we further split with $\psi^{y^{\prime} x^{\prime}}:=\psi_{x^{\prime} y^{\prime}}^{-1}$

$$
\begin{aligned}
(x-a)^{t} \psi_{x y}(y-b)= & \left(\left(x^{\prime}-a^{\prime}\right)^{t}+\left(x_{d}-a_{d}\right) \psi_{x_{d} y^{\prime}} \psi^{y^{\prime} x^{\prime}}\right)\left(\psi_{x^{\prime} y^{\prime}}\left(y^{\prime}-b^{\prime}\right)+\psi_{x^{\prime} y_{d}}\left(y_{d}-b_{d}\right)\right) \\
& +\left(x_{d}-a_{d}\right)\left(\psi_{x_{d} y_{d}}-\psi_{x_{d} y^{\prime}} \psi^{y^{\prime} x^{\prime}} \psi_{x^{\prime} y_{d}}\right)\left(y_{d}-b_{d}\right)
\end{aligned}
$$

where the derivatives of $\psi$ are evaluated at $(a, b)$. Note that by (2.10) the second term drops out if $b_{d}=\mathfrak{g}\left(a, b^{\prime}\right)$.

To define $B_{n}$ and $f_{n}$ we first consider for fixed $a_{d}$ the map $\sigma\left(\cdot, a_{d}\right)$ defined in a neighborhood of the origin $O_{R}^{\prime}$ of $\mathbb{R}^{d-1}$ by

$$
y^{\prime} \mapsto \sigma\left(y^{\prime}, a_{d}\right):=-\left.\psi_{x_{d} y^{\prime}} \psi^{y^{\prime} x^{\prime}}\right|_{\left(x^{\prime}, x_{d}, y^{\prime}, y_{d}\right)=\left(O_{L}^{\prime}, a_{d}, y^{\prime}, \mathfrak{g}\left(O_{L}^{\prime}, a_{d}, y^{\prime}\right)\right)} .
$$

Then $\sigma\left(O_{R}^{\prime}, 0\right)=O_{R}^{\prime}$. By the curvature condition (2.14) and (2.4), the map $\sigma\left(\cdot, a_{d}\right)$ is a diffeomorphism on a neighborhood of $O_{R}^{\prime}$, if $a_{d}$ is small; the bounds are uniform for $a_{d}$ in an open interval containing 0 . We may assume that the neighborhood of $O_{R}^{\prime}$ and its image contain the ball of radius $\varepsilon_{3}$ centered at $O_{R}^{\prime}$, whenever $\left|a_{d}\right| \leq \varepsilon_{3}$. Let $b^{\prime}(n)$ be defined by

$$
\sigma\left(b^{\prime}(n), \lambda^{-1 / 3} n_{d}\right)=\lambda^{-1 / 3} n^{\prime}
$$

and we assume that $|n| \lesssim \varepsilon \lambda^{1 / 6}$. Let

$$
b(n)=\left(b^{\prime}(n), b_{d}(n)\right):=\left(b^{\prime}(n), g\left(O_{L}^{\prime}, n_{d} \lambda^{-1 / 3}, b^{\prime}(n)\right)\right.
$$

and let $B_{n}$ be the ball of radius $\varepsilon \lambda^{-1 / 3}$ centered at $b(n)$. Define

$$
f_{n}(y)=\chi_{B_{n}}(y) e^{-i \lambda H(a(n), b(n), y)}
$$

with $H$ as in (3.12). It will be crucial to note that $\left|\operatorname{det} \psi_{x y}(x, y)\right| \lesssim \lambda^{-1 / 3}$ when $y \in B_{n}$, $x \in R_{n}$ (see (3.19) below).

It now suffices to show

$$
\left|\mathcal{T}_{\lambda} f_{n}(x)\right| \geq c \lambda^{-d / 3}, \quad \text { if } x \in R_{n} .
$$

with the positive constant $c$ independent of $\lambda, Q$ and $n$. To see (3.15) note that

$$
e^{-i \lambda \phi(x, b(n))} T_{\lambda} f_{n}(x)=\int e^{i \lambda \Psi_{n}(x, y)} \chi_{1}(x, y) \chi_{B_{n}}(y) d y
$$


where, by (3.13), $\Psi_{n}(x, y)=(x-a)^{t} \psi_{x y}(a, b)(y-b)+O\left(\varepsilon \lambda^{-1}\right)$ evaluated at $(a, b)=$ $(a(n), b(n))$, and the error bounds hold if $|y-a(n)| \leq \varepsilon \lambda^{-1 / 3}$, and $|x-a(n)| \leq \lambda^{-1 / 3}$. Thus estimate (3.15) follows if we verify that

$$
\left|(x-a(n))^{t} \psi_{x y}(a(n), b(n))(y-b(n))\right| \leq C \varepsilon \lambda^{-1} \text {, if } x \in R_{n} .
$$

Since the vector

$$
\left(\lambda^{-1 / 3} n^{\prime}, 1\right)=-\psi_{x_{d} y^{\prime}} \psi^{y^{\prime} x^{\prime}}\left(0^{\prime}, \lambda^{-1 / 3} n_{d}, b^{\prime}(n), \mathfrak{g}\left(0, \lambda^{-1 / 3} n_{d}, b^{\prime}(n)\right)\right)
$$

is in the kernel of the orthogonal projection $\Pi_{n^{\prime}}$ we have for $x \in R_{n}$

$$
\left|x^{\prime}-a^{\prime}(n)+\left(x_{d}-a_{d}(n)\right) \psi_{x_{d} y^{\prime}} \psi^{y^{\prime} x^{\prime}}\left(0^{\prime}, \lambda^{-1 / 3} n_{d}, b^{\prime}(n), \mathfrak{g}\left(0, \lambda^{-1 / 3} n_{d}, b^{\prime}(n)\right)\right)\right| \leq C \varepsilon \lambda^{-2 / 3} .
$$

Notice that by the crucial properties (2.9) and (2.4), (2.7) the terms $\psi_{x_{d} y^{\prime} x^{\prime}}, \psi_{x_{d} y^{\prime}}$ and $\psi_{x_{d} y^{\prime} y_{d}}$ are all $O\left(\varepsilon \lambda^{-1 / 6}\right)$ in $Q_{o}$. Thus

$$
\left|\psi_{x_{d} y^{\prime}} \psi^{y^{\prime} x^{\prime}}\left(a^{\prime}, a_{d}, b^{\prime}, \mathfrak{g}\left(a, b^{\prime}\right)\right)-\psi_{x_{d} y^{\prime}} \psi^{y^{\prime} x^{\prime}}\left(0^{\prime}, a_{d}, b^{\prime}, \mathfrak{g}\left(0, a_{d}, b^{\prime}\right)\right)\right| \leq C \varepsilon \lambda^{-1 / 3} .
$$

Consequently

$$
\left|\left(\left(x^{\prime}-a^{\prime}\right)^{t}+\left(x_{d}-a_{d}\right) \psi_{x_{d} y^{\prime}} \psi^{y^{\prime} x^{\prime}}(a, b)\right)\left(\psi_{x^{\prime} y^{\prime}}\left(y^{\prime}-b^{\prime}\right)+\psi_{x^{\prime} y_{d}}(a, b)\left(y_{d}-b_{d}\right)\right)\right| \leq C \varepsilon \lambda^{-1}
$$

if $a=a(n), b=b(n)$, the derivatives are evaluated at $(a(n), b(n))$ and $x \in R_{n}$ and $y \in B_{n}$. Moreover for these choices of $a, b, x, y$

$$
\left|\left(x_{d}-a_{d}\right)\left(\psi_{x_{d} y_{d}}-\psi_{x_{d} y^{\prime}} \psi^{y^{\prime} x^{\prime}} \psi_{x^{\prime} y_{d}}\right)\left(y_{d}-b_{d}\right)\right| \leq C \varepsilon \lambda^{-1} .
$$

To see this we use that $b_{d}(n)-\mathfrak{g}\left(a(n), b^{\prime}(n)\right)=\left\langle\mathfrak{g}_{x^{\prime}}\left(O_{L}^{\prime}, a_{d}, b^{\prime}(n)\right), a^{\prime}(n)\right\rangle+O\left(\lambda^{-1 / 3}\right)$ and since by implicit differentiation using (2.8) we have $\mathfrak{g}_{x^{\prime}}=O\left(\lambda^{-1 / 6}\right)$ we see that in fact

$$
b_{d}(n)-\mathfrak{g}\left(a(n), b^{\prime}(n)\right)=O\left(\lambda^{-1 / 3}\right) .
$$

Hence

$$
\psi_{x_{d} y_{d}}-\psi_{x_{d} y^{\prime}} \psi^{y^{\prime} x^{\prime}} \psi_{x^{\prime} y_{d}}=O\left(\lambda^{-1 / 3}\right)
$$

and thus (3.18) follows. By (3.17) and (3.18) we get (3.16) and this finishes the verification of (3.15).

Remark. The reader familiar with the wave packet analysis in the context of the classical restriction problem for the Fourier transform (see for example 38]) may find it enlightening to construct Kakeya set examples of this type for the particular operator $\left.g \mapsto \widehat{g d \sigma}\right|_{R \mathbb{S}^{d}}$ discussed in the introduction. The key point here is that if $B$ is an $R^{-1 / 3}$-cap centered at a point $x_{0}$ on the equator of $\mathbb{S}^{d}$, and if $\nu \in \mathbb{S}^{d}$ lies within a distance of $O\left(R^{-1 / 3}\right)$ of the north pole, then the function $g(x)=\chi_{B}(x) e^{i R \nu \cdot x}$ is such that $|\widehat{g d \sigma}|_{R \mathbb{S}^{d}}(\xi) \mid \gtrsim R^{-d / 3} \chi_{T_{B}}(\xi)$; here $T_{B}$ is an "eccentric cap" (or "stretched cap") on $R \mathbb{S}^{d}$ of dimensions $O\left(R^{1 / 3}\right) \times \cdots \times$ $O\left(R^{1 / 3}\right) \times O\left(R^{2 / 3}\right)$, centered at $R \nu$ and with long edges in the direction $x_{0}$. In order to exploit this we let $\left\{\nu_{m}\right\}_{1 \leq m \lesssim R^{1 / 3}}$ be a sequence of equally spaced points on the curve $\left\{u=\left(u_{1}, \ldots, u_{d+1}\right) \in \mathbb{S}^{d}: u_{1}=\cdots=u_{d-1}=0\right\}$, and let $\mathbb{S}_{\nu_{m}}^{d-1}=\left\{\omega \in \mathbb{S}^{d}: \omega \cdot \nu_{m}=0\right\}$. We now choose a collection of disjoint $R^{-1 / 3}$-caps $\left\{B_{m, n}\right\}_{1 \leq m \leq R^{1 / 3}, 1 \leq n \leq R^{(d-1) / 3}}$ on $\mathbb{S}^{d}$ such that for each $m$ and $n$ the center of $B_{m, n}$ (which we will call $x_{m, n}$ ) lies on the great sphere $\mathbb{S}_{\nu_{m}}^{d-1}$. Now, for each $m$ and $n$ let $T_{m, n}$ denote an eccentric cap on $R \mathbb{S}^{d}$ of dimensions $O\left(R^{1 / 3}\right) \times \cdots \times O\left(R^{1 / 3}\right) \times O\left(R^{2 / 3}\right)$, with long sides pointing in the direction $x_{m, n}$ and centered at a point $R \nu_{m, n} \in R \mathbb{S}^{d}$ with $\left|\nu_{m, n}-\nu_{m}\right| \lesssim R^{-1 / 3}$. Now if $g_{m, n}(x)=e^{i R \nu_{m, n} \cdot x} \chi_{B_{m, n}}(x)$ then $\left|\widehat{g_{m, n} d \sigma}\right|_{R \mathbb{S}^{d}}(\xi) \mid \gtrsim R^{-d / 3} \chi_{T_{m, n}}(\xi)$ uniformly in $m$ and $n$. Choosing the caps $B_{m, n}$ and 
frequencies $\nu_{m, n}$ appropriately, taking $g$ to be a random combination of the form $\sum \pm g_{m, n}$ and invoking appropriate Besicovitch type estimates now leads to the required necessary condition $q>(2 d+1) / d$. Here of course the $O\left(R^{1 / 3}\right)$ scaled Kakeya sets that feature are subsets of $R \mathbb{S}^{d}$ rather than $\mathbb{R}^{d}$. Notice also that an analogue of the additional decomposition at scale $O\left(\lambda^{-1 / 6}\right)$, required in the treatment of the general operators $T_{\lambda}$, is not necessary here.

\section{BASIC DECOMPOSITIONS}

It is standard to decompose the operator $T_{\lambda}$ in terms of the size of det $\phi_{x y}$. By a Taylor expansion (using (2.12), (2.5)) we observe that on the (small) support of our cutoff function

$$
y_{d}-g\left(x, y^{\prime}\right)=\mathcal{C}(x, y) \operatorname{det} \phi_{x y}
$$

with $\mathcal{C}(x, y) \neq 0$ so that the decomposition in terms of $\operatorname{det} \phi_{x y}$ can be realized by decomposing in terms of the size of $y_{d}-g\left(x, y^{\prime}\right)$. Thus we split $T_{\lambda}=\sum_{2^{l}<\lambda^{1 / 3}} T_{\lambda, l}+\widetilde{T}_{\lambda}$ where

$$
T_{\lambda, l} f(x)=\int e^{i \lambda \phi(x, y)} \chi(x, y) \chi_{1}\left(2^{l}\left(y_{d}-g\left(x, y^{\prime}\right)\right)\right) f(y) d y
$$

where $\chi_{1}$ is supported in $(2 / 3,3 / 2) \cup\left((-3 / 2,-2 / 3)\right.$ and $\widetilde{T}_{\lambda}$ is defined similarly with a cutoff $\chi_{0}\left(2^{l}\left(y_{d}-g\left(x, y^{\prime}\right)\right)\right.$ localizing to the region $\left|y_{d}-g\left(x, y^{\prime}\right)\right| \lesssim \lambda^{-1 / 3}$. Then $T_{\lambda, l}$ and $\widetilde{T}_{\lambda}$ cover the situations where $\left|\operatorname{det} \phi_{x y}\right| \approx 2^{-l}$, and $\left|\operatorname{det} \phi_{x y}\right| \lesssim \lambda^{-1 / 3}$, respectively.

By standard $L^{2}$ theory [17], 22] (see also [32] for earlier results in special cases) we have

$$
\begin{aligned}
\left\|T_{\lambda, l}\right\|_{L^{2} \rightarrow L^{2}} & \lesssim 2^{l / 2} \lambda^{-d / 2}, \quad 2^{l}<\lambda^{1 / 3}, \\
\left\|\widetilde{T}_{\lambda}\right\|_{L^{2} \rightarrow L^{2}} & \lesssim \lambda^{-(d-1) / 2-1 / 3} .
\end{aligned}
$$

Our main estimates in two dimensions are

$$
\begin{aligned}
\left\|T_{\lambda, l}\right\|_{L^{4}\left(\mathbb{R}^{2}\right) \rightarrow L^{4}\left(\mathbb{R}^{2}\right)} & \lesssim 2^{-3 l / 4} \lambda^{-1 / 2}(\log \lambda)^{1 / 4}, \quad 2^{l}<\lambda^{1 / 3}, \\
\left\|\widetilde{T}_{\lambda}\right\|_{L^{4}\left(\mathbb{R}^{2}\right) \rightarrow L^{4}\left(\mathbb{R}^{2}\right)} & \lesssim \lambda^{-3 / 4}(\log \lambda)^{1 / 4},
\end{aligned}
$$

and for $2 \leq p<4, q=3 p^{\prime}$,

$$
\begin{aligned}
\left\|T_{\lambda, l}\right\|_{L^{p}\left(\mathbb{R}^{2}\right) \rightarrow L^{q}\left(\mathbb{R}^{2}\right)} & \lesssim 2^{-l / p^{\prime}} \lambda^{-2 / q}, \quad 2^{l}<\lambda^{1 / 3}, \\
\left\|\widetilde{T}_{\lambda}\right\|_{L^{p}\left(\mathbb{R}^{2}\right) \rightarrow L^{q}\left(\mathbb{R}^{2}\right)} & \lesssim \lambda^{-1 /\left(3 p^{\prime}\right)-2 / q} .
\end{aligned}
$$

Notice that (4.6), (4.8) are limiting cases of (4.5) and (4.7). We shall prove only (4.5) and (4.7) and the proofs of (4.6) and (4.8) are analogous. Indeed for the proofs of (4.6), (4.8) the localization to the region where $\left|y_{2}-g\left(x, y_{1}\right)\right| \approx 2^{-l}$ can be replaced by the localization to the region where $\left|y_{2}-g\left(x, y_{1}\right)\right| \lesssim 2^{-l}$.

By interpolation it follows from (4.4) (with $d=2$ ), and (4.6) that

$$
\begin{aligned}
& \left\|\widetilde{T}_{\lambda}\right\|_{L^{5 / 2}\left(\mathbb{R}^{2}\right) \rightarrow L^{5 / 2}\left(\mathbb{R}^{2}\right)} \lesssim \lambda^{-4 / 5}(\log \lambda)^{1 / 10} \\
& \left\|\widetilde{T}_{\lambda}\right\|_{L^{p}\left(\mathbb{R}^{2}\right) \rightarrow L^{q}\left(\mathbb{R}^{2}\right)} \lesssim \lambda^{-2 / q}, \quad q=\frac{3 p^{\prime}}{2}, \quad q>\frac{5}{2} .
\end{aligned}
$$


Moreover the restricted weak type estimates

$$
\begin{array}{r}
\left\|\sum_{2^{l} \leq \lambda^{1 / 3}} T_{\lambda, l}\right\|_{L^{5 / 2,1}\left(\mathbb{R}^{2}\right) \rightarrow L^{5 / 2, \infty}\left(\mathbb{R}^{2}\right)} \lesssim \lambda^{-4 / 5}(\log \lambda)^{1 / 10}, \\
\left\|\sum_{2^{l} \leq \lambda^{1 / 3}} T_{\lambda, l}\right\|_{L^{p, 1}\left(\mathbb{R}^{2}\right) \rightarrow L^{q, \infty}\left(\mathbb{R}^{2}\right)} \lesssim \lambda^{-2 / q}, \quad q=\frac{3 p^{\prime}}{2}, \quad \frac{5}{2}<q \leq 3,
\end{array}
$$

follow from (4.3) and (4.5) by a now standard interpolation argument due to Bourgain [7] (see also the appendix in [11). Of course (4.11) and (4.9) imply (1.17). By a further interpolation (by the real method) we can upgrade (4.12) to

$$
\left\|\sum_{2^{l} \leq \lambda^{1 / 3}} T_{\lambda, l}\right\|_{L^{p, q}\left(\mathbb{R}^{2}\right) \rightarrow L^{q}\left(\mathbb{R}^{2}\right)} \lesssim \lambda^{-2 / q}, \quad q=\frac{3 p^{\prime}}{2}, \quad \frac{5}{2}<q<3,
$$

which implies the analogous $L^{p} \rightarrow L^{q}$ inequality, and we obtain (1.15), in the range $5 / 2<$ $q<3$. We note that the case $q \geq 3$ (corresponding to $p \leq 2$ ) is already covered by the result in 21]. Finally the inequality (1.16) follows by interpolation between the $L^{2}\left(\mathbb{R}^{2}\right)$ bound $\left\|T_{\lambda}\right\|_{L^{2} \rightarrow L^{2}}=O\left(\lambda^{-5 / 6}\right)$ and the restricted weak type estimate (1.17).

\section{BOUNDS FOR MODEL CASES}

Consider the phase function defined in $\mathbb{R}^{d}$,

$$
\phi(x, y)=\sum_{j=1}^{d-1} x_{j} y_{j}+\frac{\left(x_{d}-y_{d}\right)^{3}}{6}+x_{d} \sum_{k=1}^{d-1} y_{k}^{2},
$$

and let $\chi \in C_{0}^{\infty}\left(\mathbb{R}^{d} \times \mathbb{R}^{d}\right)$ be supported near the origin.

We observe that rank $\phi_{x y}^{\prime \prime}=d-1$, $\operatorname{det} \phi_{x y}^{\prime \prime}=x_{d}-y_{d}$, and for $x_{d}=y_{d}$ the kernel of $d \pi_{L}$ is generated by $\partial / \partial y_{d}$ and the kernel of $d \pi_{R}$ is generated by $\partial / \partial x_{d}$. Condition (1.7) is satisfied since $\phi_{x_{d} y_{d} y_{d}}=-1$ and condition (1.8) is satisfied since $\phi_{x_{d} y_{d} x_{d}}=1$. For each $x$ the hypersurface $\mathcal{L}_{x}=\left\{\phi_{x}^{\prime}(x, y): \operatorname{det} \phi_{x y}=0\right\}$ is just the paraboloid $\left\{\left(y^{\prime},\left|y^{\prime}\right|^{2}\right)\right\}$; thus condition (1.12) is satisfied.

Consider the operator $T_{\lambda, l}$ given by the localization to the set $\left\{\left|x_{d}-y_{d}\right| \approx 2^{-l}\right\}$. We now split $f=\sum f_{m}$ where $f_{m}(y)=\chi_{I_{m, l}}\left(y_{d}\right) f(y)$ and $I_{m, l}=\left[m 2^{-l},(m+1) 2^{-l}\right]$. Then $T_{\lambda, l} f_{m}(x)$ vanishes if $x_{d} \notin I_{m, l}^{*}:=I_{m-1, l} \cup I_{m, l} \cup I_{m+1, l}$. Thus

$$
\left\|T_{\lambda, l} f\right\|_{q} \leq C\left(\sum_{m}\left\|T_{\lambda, l} f_{m}\right\|_{q}^{q}\right)^{1 / q}
$$

and so it suffices to estimate $T_{\lambda, l} f_{m}$. Now we write

$$
T_{\lambda, l} f_{m}=\int_{I_{m, l}} e^{i \lambda\left(x_{d}-y_{d}\right)^{3} / 6} T_{\lambda, l, y_{d}}\left[f_{m}\left(\cdot, y_{d}\right)\right] d y_{d}
$$

where for $g$ being defined on $\mathbb{R}^{d-1}$,

$$
T_{\lambda, l, y_{d}} g\left(x^{\prime}, x_{d}\right)=\int e^{i \lambda \Psi\left(x, y^{\prime}\right)} \chi\left(x, y^{\prime}, y_{d}\right) g\left(y^{\prime}\right) d y^{\prime}
$$

and

$$
\Psi\left(x, y^{\prime}\right)=\sum_{j=1}^{d-1} x_{j} y_{j}+x_{d} \sum_{k=1}^{d-1} y_{k}^{2}
$$


Now let $d=2$. The phase function $\Psi$ is such that we can apply the Fefferman-Stein adjoint restriction theorem ([20]), or the more general Carleson-Sjölin theorem ([14], 24]) and obtain the estimates

$$
\left\|T_{\lambda, l, y_{2}} g\right\|_{L^{q}\left(\mathbb{R}^{2}\right)} \lesssim \lambda^{-2 / q}\|g\|_{L^{p}(\mathbb{R})}, \quad q=3 p^{\prime}, p<4 ;
$$

uniformly in $y_{2} \in I_{m, l}$. Then

$$
\begin{aligned}
\left\|T_{\lambda, l} f_{m}\right\|_{L^{q}\left(\mathbb{R}^{2}\right)} \lesssim & \int_{I_{m, l}}\left\|T_{\lambda, l, y_{2}}\left[f_{m}\left(\cdot, y_{2}\right)\right]\right\|_{L^{q}\left(\mathbb{R}^{2}\right)} d y_{2} \lesssim \lambda^{-2 / q} \int_{I_{m, l}}\left\|f_{m}\left(\cdot, y_{2}\right)\right\|_{L^{p}(\mathbb{R})} d y_{2} \\
& \lesssim 2^{-l / p^{\prime}} \lambda^{-2 / q}\left(\int_{I_{m, l}}\left\|f_{m}\left(\cdot, y_{2}\right)\right\|_{L^{p}\left(\mathbb{R}^{2}\right)}^{p} d y_{2}\right)^{1 / p} \lesssim 2^{-l / p^{\prime}} \lambda^{-2 / q}\left\|f_{m}\right\|_{L^{p}\left(\mathbb{R}^{2}\right)},
\end{aligned}
$$

and (4.7) is now implied by (5.2). The estimate (4.8) follows in a similar way. Moreover the bounds (4.5) and (4.6) follow by using an endpoint $L^{4}$ bound of the Carleson-Sjölin theorem.

Higher dimensions. A similar argument gives also a partial result in higher dimensions. Namely, for the operator with model phase (5.1) there is the bound

$$
\left\|T_{\lambda}\right\|_{L^{p}\left(\mathbb{R}^{d}\right) \rightarrow L^{q}\left(\mathbb{R}^{d}\right)} \leq \lambda^{-d / q}, \quad q \geq \frac{d+1}{d} p^{\prime}, \quad q>\frac{2\left(d^{2}+d-1\right)}{d^{2}} .
$$

The range $q \geq 2(d+1) / d$ is covered by [21], and for $2\left(d^{2}+d-1\right) / d^{2}<q<2(d+1) / d$ one can use Tao's adjoint restriction estimate for paraboloids [38. Indeed this estimate implies that the $L^{r}\left(\mathbb{R}^{d-1}\right) \rightarrow L^{s}\left(\mathbb{R}^{d}\right)$ operator norm of $T_{\lambda, l, y_{d}}$ is $O\left(\lambda^{-d / s}\right)$, provided that $s=\frac{d+1}{d-1} r^{\prime}$ and $s>2(d+2) / d$. By the above argument using Hölder's inequality in the $y_{d}$ variable

$$
\left\|T_{\lambda, l} f\right\|_{s} \lesssim 2^{-l / r^{\prime}} \lambda^{-d / s}\|f\|_{r}, \quad s=\frac{d+1}{d-1} r^{\prime}, \quad \frac{2(d+2)}{d}<s<\frac{2(d+1)}{d-1} .
$$

We also use the $L^{2} \rightarrow L^{2}$ bound (4.3) and Bourgain's interpolation lemma. One deduces that the operators $\sum_{2^{l}<\lambda^{1 / 3}} T_{\lambda, l}$ map $L^{p, 1}$ to $L^{q, \infty}$ with norm $O\left(\lambda^{-d / q}\right)$ if $q \leq(d+1) p^{\prime} / d$ and $q>2\left(d^{2}+d-1\right) / d^{2}$. By a further interpolation the strong type $L^{p} \rightarrow L^{q}$ bound now follows in the same range; moreover there are similar bounds for $\widetilde{T}_{\lambda}$. Hence one obtains (5.4) in the full range.

We conjecture that this behavior remains true for general oscillatory integral operators with folding canonical relations, satisfying the elliptical curvature condition (1.12). Wellknown (hyperbolic) examples of Bourgain in [9] may be adapted to show that an ellipticity condition is in fact necessary here. We hope to pursue these questions in a subsequent paper.

It is conjectured that the oscillatory integral operator $S_{\lambda}$ associated to the Carleson-Sjölin model phase $\Psi\left(x, y^{\prime}\right)$ as in (5.3) has an $L^{r}\left(\mathbb{R}^{d-1}\right) \rightarrow L^{s}\left(\mathbb{R}^{d}\right)$ operator norm $O\left(\lambda^{-d / s}\right)$ for $s \geq \frac{d+1}{d-1} r^{\prime}, r<2 d /(d-1)$. The above analysis suggests that the bound (5.4) for the model case might be valid in the range $q>(2 d+1) / d$. Note that $2\left(d^{2}+d-1\right) / d^{2} \geq(2 d+1) / d$ for $d \geq 2$, with equality only for $d=2$.

One-sided fold conditions. Examples suggest that the $L^{p} \rightarrow L^{q}$ estimates in Theorem 1.2 for $p>5 / 2$ may hold merely under the one-sided assumption (1.7) and the curvature condition (1.12). This is in contrast to the $L^{2}$ estimates where the bounds depend on finite type conditions on the projection $\pi_{R}$, see [21, [16] and also the survey [23]. 
A simple example (where $\pi_{R}$ is maximally degenerate) is given by

$$
\psi(x, y)=\sum_{j=1}^{d-1} x_{j} y_{j}+x_{d} y_{d}^{2}+x_{d} \sum_{k=1}^{d-1} y_{k}^{2} .
$$

Now det $\psi_{x y}=2 y_{d}$ and a simple modification of the above slicing argument shows that the $L^{p} \rightarrow L^{q}$ operator norm of the corresponding oscillatory integral operator is again $O\left(\lambda^{-d / q}\right)$ if $q \geq(d+1) p^{\prime} / d$ and $q>2\left(d^{2}+d-1\right) / d^{2}$. It would be interesting to know whether this result holds in general under merely the assumptions (1.7), (1.12). In 21] it had been shown that this is the case in the range $q \geq 2(d+1) / d$.

\section{Estimation of $T_{\lambda, l}$ IN TWO Dimensions}

We shall now fix $l$ and various decompositions will depend on $l$ but this will not be indicated. We shall estimate the square of $T_{\lambda, l} f$ and bilinearize the problem as follows. We split

$$
\left(T_{\lambda, l} f\right)^{2}=\sum_{m \geq 0} \mathfrak{B}^{m}(f, f)
$$

where

$$
\mathfrak{B}^{0}(f, \tilde{f})(x)=\iint \chi_{0}\left(2^{-5+l / 2}\left(y_{1}-\tilde{y}_{1}\right)\right) e^{i \lambda(\phi(x, y)+\phi(x, \tilde{y}))} \zeta_{l}(x, y) \zeta_{l}(x, \tilde{y}) f(y) \tilde{f}(\tilde{y}) d y d \tilde{y},
$$

and, for $m>0$,

$$
\mathfrak{B}^{m}(f, \tilde{f})(x)=\iint \chi_{1}\left(2^{l / 2-m-5}\left(y_{1}-\tilde{y}_{1}\right)\right) e^{i \lambda(\phi(x, y)+\phi(x, \tilde{y}))} \zeta_{l}(x, y) \zeta_{l}(x, \tilde{y}) f(y) \tilde{f}(\tilde{y}) d y d \tilde{y},
$$

and

$$
\zeta_{l}(x, y)=\chi(x, y) \chi_{1}\left(2^{l}\left(y_{2}-g\left(x, y_{1}\right)\right)\right) .
$$

Notice that the sum in $m$ is extended over those $m \geq 0$ with $m<l / 2-C$ for large $C$ in view of the smallness of the support of the cutoff function.

We shall show that

$$
\left\|\mathfrak{B}^{0}(f, \tilde{f})\right\|_{q / 2}+\left\|\sum_{m>0} \mathfrak{B}^{m}(f, \tilde{f})\right\|_{q / 2} \lesssim 2^{-2 l / p^{\prime}} \lambda^{-4 / q}\|f\|_{p}\|\tilde{f}\|_{p} \quad \text { for } 1 \leq p<4, q=3 p^{\prime}
$$

moreover

$$
\left\|\mathfrak{B}^{0}(f, \tilde{f})\right\|_{2} \lesssim 2^{-3 l / 2} \lambda^{-1}(\log \lambda)^{1 / 2}\|f\|_{4}\|\tilde{f}\|_{4}
$$

and

$$
\left\|\sum_{m>0} \mathfrak{B}^{m}(f, \tilde{f})\right\|_{2} \lesssim 2^{-3 l / 2}(1+l)^{1 / 2} \lambda^{-1}\|f\|_{4}\|\tilde{f}\|_{4}
$$

In what follows we shall estimate the expression $\sum_{m>0} \mathfrak{B}^{m}(f, f)$ for $m>0$ and give the modifications for $\mathfrak{B}^{0}(f, f)$ in 8

The principal objective of our approach is to reduce matters to an $L^{2}$ estimate for some well-localized operators (termed $\mathfrak{S} \equiv \mathfrak{S}_{a \nu}^{m \mu b \tilde{b}}$ below), for which one can use arguments for model cases considered in $\$ 5$. The idea is to estimate such a localized operator $\mathfrak{S}$, by freezing the variables $y_{2}, \widetilde{y}_{2}$, and to take advantage of the small support by using Hölder's 
inequality. It was possible to implement this idea "globally" in "rigid" model cases, such as $g\left(x, y_{1}\right)=x_{2}+x_{1}-y_{1}$, but this global approach does not seem to work for general $g$.

It seems natural to decompose for fixed $x$ the set $\left\{y:\left|y_{2}-g\left(x, y_{1}\right)\right| \approx 2^{-l}\right\}$ into rectangular pieces of size $\approx 2^{-l / 2} \times 2^{-l}$. In order to be useful this decomposition should be stable under perturbations in $x$ but since $g\left(x, y_{1}\right)$ varies in $x$ we need a decomposition in $x$ as well. For a situation in which we can use the idea of freezing $y_{2}$ we may consider the case that $y_{1}, \tilde{y}_{1}$ are supported in intervals $I, \widetilde{I}$ of length $2^{-l / 2}$ and $x$ is localized to certain rectangles $R$ of size $2^{-l / 2} \times 2^{-l}$ so that for $\left(x, y_{1}, \widetilde{y}_{1}\right) \in R \times I \times \widetilde{I}$ the expressions $g\left(x, y_{1}\right)$ and $g\left(x, \tilde{y}_{1}\right)$ vary by no more than $2^{-l}$. This works well if the distance of $I$ and $\widetilde{I}$ is not much more than their length, namely $2^{-l / 2}$. The rectangles in $x$-space are not supposed to change orientation while their centers vary over a cube of sidelength $2^{-l / 2}$ and the geometry of $\mathcal{L}$ suggests that the long sides become perpendicular to $\nabla_{x} g$ at the centers of the cubes.

When estimating the $\mathfrak{B}^{m}(f, \widetilde{f})$ we are in the situation where $\left|y_{1}-\widetilde{y}_{1}\right| \approx 2^{-l / 2+m+5}$ for $0<m<l / 2$. We would then like to make a similar decomposition of intervals $I \times \widetilde{I}$ in $\left(y_{1}, \widetilde{y}_{1}\right)$ space and rectangles $R$ in $x$ space. The requirement that both expressions $g\left(x, y_{1}\right)$ and $g\left(x, \tilde{y}_{1}\right)$ vary by no more than $2^{-l}$ in $R \times I \times \widetilde{I}$ is now harder to satisfy and we need to choose a finer decomposition, namely we choose the intervals $I, \widetilde{I}$ to be of length $2^{-l / 2-m}$ (cf. (6.8) below) and make a decomposition in terms in $x$ into cubes of the sidelength $2^{-l / 2-m}$ (cf. (6.6) below). This is somewhat reminiscent of a situation in 34. Moreover we decompose each cube in $x$ space into smaller rectangles of sidelengths $2^{-l / 2-m}$ and $2^{-l}$ and the longer sides are perpendicular to $\nabla_{x} g$ at the centers of the cubes ( $c f$. (6.7) below). The geometry is now such that the orientation of the rectangles is essentially the same when $y_{1}$ varies over $I, \widetilde{y}_{1}$ varies over $\widetilde{I}$ and $x$ varies over $R$. Note that the rectangles become essentially cubes of length $2^{-l}$ when the distance of the intervals is $\approx 1$; in this case the length of the intervals $I, \widetilde{I}$ is also $\approx 2^{-l}$.

We now formally define these decompositions in the $x$ and $y$ variables. Let $\eta$ be a $C^{\infty}$ function supported in $(-3 / 4,3 / 4)$ so that $\sum_{n \in \mathbb{Z}} \eta^{2}(s-n) \equiv 1$. For each $b \in 2^{-l / 2-m} \mathbb{Z}$ let $J_{b}^{m}$ be the interval of length $2^{-l / 2-m+1}$ centered at $b$. We let $\mathcal{P}^{m}$ be the set of all pairs $(b, \tilde{b})$ with the property that $J_{b}^{m} \times J_{\tilde{b}}^{m}$ intersects the support of the cutoff function $\left(y_{1}, \tilde{y}_{1}\right) \mapsto \chi_{1}\left(2^{l / 2-m-5}\left|y_{1}-\tilde{y}_{1}\right|\right)$, so that for $(b, \tilde{b}) \in \mathcal{P}^{m}$ the numbers $b, \tilde{b}$ are $C 2^{-l / 2+m_{-}}$ separated. We may split $\mathcal{P}^{m}=\cup_{\mu} \mathcal{P}_{\mu}^{m}$ where $\mu$ ranges over $2^{-l / 2+m} \mathbb{Z}$, the families $\mathcal{P}_{\mu}^{m}$ are disjoint and of cardinality $O\left(2^{4 m}\right)$ and we have

$$
|b-\mu| \leq 2^{-l / 2+m+1}, \quad|\tilde{b}-\mu| \leq 2^{-l / 2+m+1}, \quad \text { for }(b, \tilde{b}) \in \mathcal{P}_{\mu}^{m} .
$$

We also decompose in $x$ space, using two parameters $a, \nu$. The parameter $a$ will range over points in $2^{-l / 2-m} \mathbb{Z}^{2}$. For $\mu \in 2^{-l / 2+m} \mathbb{Z}$ and $\nu \in \mathbb{Z}$ (typically $|\nu| \leq C 2^{l / 2-m}$ ) we set

$$
\tau_{\nu}^{\mu} a:=a+2^{-l} \nu \frac{\nabla_{x} g(a, \mu)}{\left|\nabla_{x} g(a, \mu)\right|}
$$

and

$$
\begin{aligned}
u_{a}^{m}(x) & :=\eta\left(2^{l / 2+m}(x-a)\right) \\
u_{a \nu}^{m \mu}(x) & :=\eta\left(2^{l / 2+m}(x-a)\right) \eta\left(2^{l}\left(\left\langle\frac{\nabla_{x} g(a, \mu)}{\left|\nabla_{x} g(a, \mu)\right|}, x-\tau_{\nu}^{\mu} a\right\rangle\right)\right) .
\end{aligned}
$$


Moreover for $(b, \tilde{b}) \in \mathcal{P}_{\mu}^{m}$, we make the following definitions. Let $\beta_{0} \in C_{0}^{\infty}(\mathbb{R})$ be equal to 1 near 0 so that $\chi_{1} \beta_{0}=\chi_{1}$. Set

$$
\begin{aligned}
\omega_{a}^{m b}(y) & =\eta\left(2^{l / 2+m}\left(y_{1}-b\right)\right) \beta_{0}\left(C^{-1} 2^{l / 2+m}\left(y_{2}-g\left(a, y_{1}\right)\right)\right) \\
\omega_{a \nu}^{m \mu b}(y) & :=\omega_{a}^{m b}(y) \beta_{0}\left(C^{-1} 2^{l}\left(y_{2}-g\left(\tau_{\nu}^{\mu} a, y_{1}\right)\right)\right)
\end{aligned}
$$

and finally

$$
\Omega_{a}^{m b \tilde{b}}(y, \tilde{y}):=\omega_{a}^{m b}(y) \omega_{a}^{m \tilde{b}}(\tilde{y}), \quad \Omega_{a \nu}^{m \mu b \tilde{b}}(y, \tilde{y}):=\omega_{a \nu}^{m \mu b}(y) \omega_{a \nu}^{m \mu \tilde{b}}(\tilde{y}) .
$$

For locally integrable functions $F$ defined on $\mathbb{R}^{4}$ and $m>0$ we set

$$
\begin{aligned}
& \mathfrak{S}_{a \nu}^{m \mu b \tilde{b}} F(x)=\left(u_{a \nu}^{m \mu}(x)\right)^{2} \\
& \times \iint \chi_{1}\left(2^{l / 2-m-5}\left(y_{1}-\tilde{y}_{1}\right)\right) \zeta_{l}(x, y) \zeta_{l}(x, \tilde{y}) e^{i \lambda(\phi(x, y)+\phi(x, \tilde{y}))} \Omega_{a \nu}^{m \mu b \tilde{b}}(y, \tilde{y}) F(y, \tilde{y}) d y d \tilde{y}
\end{aligned}
$$

and for $m=0$ we use a similar definition with the modification that $\chi_{1}\left(2^{l / 2-m-5}\left(y_{1}-\tilde{y}_{1}\right)\right)$ is replaced by $\chi_{0}\left(2^{l / 2-5}\left(y_{1}-\tilde{y}_{1}\right)\right)$.

Typically the operator $\mathfrak{S}_{a \nu}^{m \mu b \tilde{b}}$ should be acting on the function

$$
(y, \tilde{y}) \mapsto f_{a \nu}^{m \mu b} \otimes \tilde{f}_{a \nu}^{m \mu \tilde{b}}(y, \tilde{y}):=\omega_{a \nu}^{m \mu b}(y) f(y) \omega_{a \nu}^{m \mu \tilde{b}}(\tilde{y}) \tilde{f}(\tilde{y})
$$

when $(b, \tilde{b}) \in \mathcal{P}_{\mu}^{m}$. Indeed, in view of the condition $\sum_{n \in \mathbb{Z}} \eta^{2}(s-n) \equiv 1$, we have

$$
\mathfrak{B}^{m}(f, f)=\sum_{a, \nu} \sum_{\mu} \sum_{(b, \tilde{b}) \in \mathcal{P}_{\mu}^{m}} \mathfrak{S}_{a \nu}^{m \mu b \tilde{b}}\left(f_{a \nu}^{m \mu b} \otimes \tilde{f}_{a \nu}^{m \mu \tilde{b}}\right) .
$$

In 97 we shall prove the following inequality concerning vector-valued functions, which combines various orthogonality arguments with the individual estimates for the operators $\mathfrak{S}_{a \nu}^{m \mu b \tilde{b}}$. As we shall see, the proof relies on ideas related to the Carleson-Sjölin theorem.

Proposition 6.1. For $2 \leq r \leq \infty$

$$
\begin{aligned}
& \left\|\sum_{0<m<l / 2} \sum_{\mu \in 2^{-l / 2+m}} \sum_{\mathbb{Z}(b, \tilde{b}) \in \mathcal{P}_{\mu}^{m}} \sum_{a, \nu} \mathfrak{S}_{a \nu}^{m \mu b \tilde{b}} F_{a \nu}^{m \mu b \tilde{b}}\right\|_{r} \lesssim \\
& \lambda^{-2 / r}\left(\sum_{0<m<l / 2} 2^{-(m+3 l / 2) \frac{r^{\prime}}{r}} \sum_{\mu} \sum_{(b, \tilde{b}) \in \mathcal{P}_{\mu}^{m}}\left(\sum_{a, \nu}\left(\iint\left|F_{a \nu}^{m \mu b \tilde{b}}(y, \tilde{y})\right|^{r^{\prime}} d y d \tilde{y}\right)^{\frac{r}{r^{\prime}}}\right)^{\frac{r^{\prime}}{r}}\right)^{\frac{1}{r^{\prime}}} .
\end{aligned}
$$

We shall have to choose the functions $F_{a \nu}^{m \mu b \tilde{b}}$ carefully in order to take full advantage of Proposition 6.1. As mentioned above we would like to let $\mathfrak{S}_{a \nu}^{m \mu b \tilde{b}}$ act on the function (6.12). However we shall have to exploit finer frequency localization properties of the operator $\mathfrak{S}_{a \nu}^{m \mu b \tilde{b}}$. Split

$$
f_{a \nu}^{m \mu b}=\mathcal{L} f_{a \nu}^{m \mu b}(y)+\mathcal{E} f_{a \nu}^{m \mu b}(y)
$$

where

$$
\mathcal{L} f_{a \nu}^{m \mu b}(y)=\omega_{a \nu}^{m \mu b}(y) \int f\left(z_{1}, y_{2}\right) \omega_{a}^{m b}\left(z_{1}, y_{2}\right) \int \beta_{2}\left(\frac{\lambda \phi_{y_{1}}(a, b, g(a, b))+\eta}{\lambda 2^{-l / 2-m}}\right) e^{i \eta\left(y_{1}-z_{1}\right)} \frac{d \eta}{2 \pi} d z_{1}
$$


and

$$
\mathcal{E} f_{a \nu}^{m \mu b}(y)=\omega_{a \nu}^{m \mu b}(y) \int f\left(z_{1}, y_{2}\right) \omega_{a}^{m b}\left(z_{1}, y_{2}\right) \int\left(1-\beta_{2}\left(\frac{\lambda \phi_{y_{1}}(a, b, g(a, b))+\eta}{\lambda 2^{-l / 2-m}}\right)\right) e^{i \eta\left(y_{1}-z_{1}\right)} \frac{d \eta}{2 \pi} d z_{1}
$$

here the function $\beta_{2}$ is supported in the union of $\left(-2 C_{0},-\left(2 C_{0}\right)^{-1}\right)$ and $\left(\left(2 C_{0}\right)^{-1}, 2 C_{0}\right)$ and $\beta_{2}(s)=1$ if $|s| \in\left[C_{0}^{-1}, C_{0}\right]$, for suitably large $C_{0}$, and the integral in the definition of $\mathcal{E} f_{a \nu}^{m \tilde{b}}$ is to be interpreted as an oscillatory integral. Now

$$
\mathfrak{S}_{a \nu}^{m \mu \nu \tilde{b}}\left(f_{a \nu}^{m \mu b} \otimes \tilde{f}_{a \nu}^{m \mu \tilde{b}}\right)=\mathfrak{S}_{a \nu}^{m \mu b \tilde{b}}\left(\left(\mathcal{L} f_{a \nu}^{m \mu b}+\mathcal{E} f_{a \nu}^{m \mu b}\right) \otimes\left(\mathcal{L} \tilde{f}_{a \nu}^{m \mu \tilde{b}}+\mathcal{E} \tilde{f}_{a \nu}^{m \mu \tilde{b}}\right)\right)
$$

but only the contribution of $\mathcal{L} f_{a \nu}^{m \mu b} \otimes \mathcal{L} \tilde{f}_{a \nu}^{m \mu \tilde{b}}$ is relevant:

Lemma 6.2. There are the pointwise bounds

$$
\begin{aligned}
& \mathfrak{S}_{a \nu}^{m \mu b \tilde{b}}\left(\mathcal{E} f_{a \nu}^{m \mu b} \otimes \mathcal{L} \tilde{f}_{a \nu}^{m \mu \tilde{b}}\right)=O\left(\left(\lambda 2^{-2 l}\right)^{-N}\right) \\
& \mathfrak{S}_{a \nu}^{m \mu b \tilde{b}}\left(\mathcal{L} f_{a \nu}^{m \mu b} \otimes \mathcal{E} \tilde{f}_{a \nu}^{m \mu \tilde{b}}\right)=O\left(\left(\lambda 2^{-2 l}\right)^{-N}\right) \\
& \mathfrak{S}_{a \nu}^{m \mu b \tilde{b}}\left(\mathcal{E} f_{a \nu}^{m \mu b} \otimes \mathcal{E} \tilde{f}_{a \nu}^{m \mu \tilde{b}}\right)=O\left(\left(\lambda 2^{-2 l}\right)^{-N}\right)
\end{aligned}
$$

Sketch of proof. We only consider the term $\mathfrak{S}_{a \nu}^{m \mu b \tilde{b}}\left(\mathcal{E} f_{a \nu}^{m \mu b} \otimes \mathcal{L} \tilde{f}_{a \nu}^{m \mu \tilde{b}}\right)$; the others are handled similarly.

The analysis leads to the estimation of oscillatory integrals of the form

$$
\iint K\left(x, z_{1}, y, \tilde{z}_{1}, y\right) e^{i\left(\lambda \phi\left(x, y_{1}, y_{2}\right)+\eta y_{1}\right)}\left(1-\beta_{2}\left(\frac{\lambda \phi_{y_{1}}^{\prime}(a, b, g(a, b))+\eta}{\lambda 2^{-l / 2-m}}\right)\right) d y_{1} d \eta
$$

where $K$ is a function satisfying $\partial_{y_{1}}^{\alpha} K=O\left(2^{\alpha l}\right)$ which vanishes for $|x-a| \gtrsim 2^{-m-l / 2}$, $\left|y_{1}-b_{1}\right| \gtrsim 2^{-m-l / 2},\left|y_{2}-g\left(x, y_{1}\right)\right| \gtrsim 2^{-l}$. The $y_{1}$ derivative of the phase is then

$$
\lambda \phi_{y_{1}}\left(x, y_{1}, y_{2}\right)+\eta=\lambda \phi_{y_{1}}^{\prime}(a, b, g(a, b))+\eta+O\left(\lambda 2^{-l / 2-m}\right) .
$$

As we assume the constant $C_{0}$ in the definition of the cutoff function $\beta_{2}$ to be large we see that

$$
\left|\lambda \phi_{y_{1}}\left(x, y_{1}, y_{2}\right)+\eta\right| \gtrsim\left|\lambda \phi_{y_{1}}^{\prime}(a, b, g(a, b))+\eta\right| \gg \lambda 2^{-l / 2-m} .
$$

The assertion then follows by an integration by parts with respect to the $y_{1}$ variable.

We shall need an orthogonality property of the $\mathcal{L} f_{a \nu}^{m \mu b}$. Set

$$
f^{m b}(y)=\chi_{\left[b-2^{-l / 2-m}, b+2^{-l / 2-m}\right]}\left(y_{1}\right) f(y) .
$$

Lemma 6.3. For $p \geq 2$ and fixed $m, b, \mu$

$$
\left(\sum_{a, \nu}\left\|\mathcal{L} f_{a \nu}^{m \mu b}\right\|_{p}^{p}\right)^{1 / p} \lesssim\left\|f^{m b}\right\|_{p}
$$

uniformly in $m, \mu, b$.

Proof. First note that for fixed $m, \mu, b, a$ the supports of the functions

$$
\beta_{0}\left(C^{-1} 2^{l / 2}\left(y_{2}-g\left(\tau_{\nu}^{\mu} a, y_{1}\right)\right)\right)
$$

have uniformly bounded overlap on the support of $\omega_{a}^{m b}$.

Define

$$
L_{a}^{m b} f(y)=\omega_{a}^{m b}(y) \int f\left(z_{1}, y_{2}\right) \omega_{a}^{m b}\left(z_{1}, y_{2}\right) \frac{1}{2 \pi} \int \beta_{2}\left(\frac{\lambda \phi_{y_{1}}^{\prime}(a, b, g(a, b))+\eta}{\lambda 2^{-l / 2-m}}\right) e^{i \eta\left(y_{1}-z_{1}\right)} d \eta d z_{1}
$$


Then the left hand side of (6.21) is dominated by a constant times the left hand side of the following inequality

$$
\left(\sum_{a}\left\|L_{a}^{m b} f^{m b}\right\|_{p}^{p}\right)^{1 / p} \lesssim\left\|f^{m b}\right\|_{p}
$$

which we now prove. It is easy to see that the operators $L_{a}^{m b}$ are uniformly bounded on $L^{\infty}$ and an interpolation argument reduces the proof of (6.23) to the case $p=2$.

In order to complete this proof it suffices to check that

$$
\left\|L_{a}^{m b}\left(L_{a^{\prime}}^{m b}\right)^{*}\right\|_{L^{2} \rightarrow L^{2}} \lesssim 2^{-\left|n-n^{\prime}\right|} \quad \text { if } a=2^{-m-l / 2} n, a^{\prime}=2^{-m-l / 2} n^{\prime},\left|n-n^{\prime}\right| \geq M,
$$

for suitably large $M$.

The kernel of $L_{a}^{m b}\left(L_{a^{\prime}}^{m b}\right)^{*}$ is given by

$$
\begin{aligned}
& \mathcal{K}\left(y, y^{\prime}\right)=\delta\left(y_{2}-y_{2}^{\prime}\right) \omega_{a}^{m b}(y) \omega_{a}^{m b}\left(y^{\prime}\right) \int \omega_{a}^{m b}\left(z_{1}, y_{2}\right) \omega_{a}^{m b}\left(z_{1}, y_{2}^{\prime}\right) \\
& \times\left\{\iint e^{i\left(\left(y_{1}-z_{1}\right) \eta-\left(y_{1}^{\prime}-z_{1}\right) \eta^{\prime}\right)} \beta_{2}\left(\frac{\lambda \phi_{y_{1}}(a, b, g(a, b))+\eta}{\lambda 2^{-l / 2-m}}\right) \beta_{2}\left(\frac{\lambda \phi_{y_{1}}\left(a^{\prime}, b, g\left(a^{\prime}, b\right)\right)+\eta^{\prime}}{\lambda 2^{-l / 2-m}}\right) \frac{d \eta}{2 \pi} \frac{d \eta^{\prime}}{2 \pi}\right\} d z_{1}
\end{aligned}
$$

We shall now fix $a^{\prime}$ and solve the equation $g\left(x_{1}, x_{2}, b\right)=g\left(a_{1}^{\prime}, a_{2}^{\prime}, b\right)$ in $x_{2}$; this can be done by the implicit function theorem since

$$
g_{x_{2}}\left(x, y_{1}\right)=-\frac{\phi_{x_{2} y_{2} x_{2}}\left(x, y_{1}, g\left(x, y_{1}\right)\right)}{\phi_{x_{2} y_{2} y_{2}}\left(x, y_{1}, g\left(x, y_{1}\right)\right)}+o(1)
$$

where $o(1)$ is a quantity which by (2.4) vanishes at the reference point $P$ and the two sided fold assumption $\left(\underline{(2.5)},(\underline{2.6})\right.$ ) implies $g_{x_{2}} \neq 0$. For later reference we also note that

$$
g_{x_{1}}\left(x, y_{1}\right)=-\frac{\phi_{x_{2} y_{2} x_{1}}\left(x, y_{1}, g\left(x, y_{1}\right)\right)}{\phi_{x_{2} y_{2} y_{2}}\left(x, y_{1}, g\left(x, y_{1}\right)\right)}+o(1)=o(1)
$$

which follows from (2.4) and (2.8).

Let thus $h\left(x_{1}, a^{\prime}, b\right)$ denote the unique solution satisfying

$$
\begin{aligned}
g\left(x_{1}, h\left(x_{1}, a^{\prime}, b\right), b\right) & =g\left(a^{\prime}, b\right), \\
h\left(a_{1}^{\prime}, a^{\prime}, b\right) & =a_{2}^{\prime} .
\end{aligned}
$$

Then

$$
\begin{aligned}
g(a, b)-g\left(a^{\prime}, b\right) & =g(a, b)-g\left(a_{1}, h\left(a_{1}, a^{\prime}, b\right), b\right)+g\left(a_{1}, h\left(a_{1}, a^{\prime}, b\right), b\right)-g\left(a^{\prime}, b\right) \\
& =\mathcal{C}\left(a, a^{\prime}, b\right)\left(a_{2}-h\left(a_{1}, a^{\prime}, b\right)\right)+O\left(a_{1}-a_{1}^{\prime}\right)
\end{aligned}
$$

where $\mathcal{C}\left(a, a^{\prime}, b\right) \neq 0$. Thus if for some small constant $c_{0}$ and some large constant $C_{0}$

$$
\left|a_{1}-a_{1}^{\prime}\right| \leq c_{0}\left|a_{2}-h\left(a_{1}, a^{\prime}, b\right)\right| \quad \text { and }\left|a_{2}-h\left(a_{1}, a^{\prime}, b\right)\right| \geq C_{0} 2^{-m-l / 2},
$$

then $\left|g(a, b)-g\left(a^{\prime}, b\right)\right| \geq C_{1} 2^{-m-l / 2}$ for still large $C_{1}$ and therefore we have

$$
L_{a}^{m b}\left(L_{a^{\prime}}^{m b}\right)^{*}=0
$$

in the case (6.29).

In the relevant opposite case we assume that

$$
\left|a_{1}-a_{1}^{\prime}\right| \geq c_{0}\left|a_{2}-h\left(a_{1}, a^{\prime}, b\right)\right| \quad \text { and }\left|a_{1}-a_{1}^{\prime}\right| \geq C_{1} 2^{-m-l / 2}
$$


Since by (6.26) we also have $h_{x_{1}}=o(1)$ it follows that in the present case (6.31) we have $\left|a_{1}-a_{1}^{\prime}\right| \gg\left|a_{2}-a_{2}^{\prime}\right|$ and therefore we can estimate with $P_{a^{\prime} b}=\left(a^{\prime}, b, g\left(a^{\prime}, b\right)\right)$

$$
\begin{aligned}
& \phi_{y_{1}}(a, b, g(a, b))-\phi_{y_{1}}\left(a^{\prime}, b, g\left(a^{\prime}, b\right)\right) \\
& =\phi_{y_{1}}(a, b, g(a, b))-\phi_{y_{1}}\left(a_{1}, h\left(a_{1}, a^{\prime}, b\right), b, g\left(a_{1}, h\left(a_{1}, a^{\prime}, b\right), b\right)\right) \\
& \quad+\phi_{y_{1}}\left(a_{1}, h\left(a_{1}, a^{\prime}, b\right), b, g\left(a_{1}, h\left(a_{1}, a^{\prime}, b\right), b\right)\right)-\phi_{y_{1}}\left(a^{\prime}, b, g\left(a^{\prime}, b\right)\right) \\
& =\phi_{x_{1} y_{1}}\left(P_{a^{\prime} b}\right)\left(a_{1}-a_{1}^{\prime}\right)+O\left(\varepsilon\left|a_{1}-a_{1}^{\prime}\right|\right)+O\left(\varepsilon\left|a_{2}-h\left(a_{1}, a^{\prime}, b\right)\right|\right)
\end{aligned}
$$

if the support of the initial cutoff function has diameter $\leq \varepsilon$. Here, in order to get the $\varepsilon$-bound, we used the assumption (2.4). Thus in case (6.31) we get

$$
\left|\phi_{y_{1}}(a, b, g(a, b))-\phi_{y_{1}}\left(a^{\prime}, b, g\left(a^{\prime}, b\right)\right)\right| \geq C\left|a_{1}-a_{1}^{\prime}\right| \approx\left|a-a^{\prime}\right| .
$$

Hence by an integration by parts in the $z_{1}$ variable we gain negative powers of

$$
2^{-l}\left|\eta-\eta^{\prime}\right| \gtrsim 2^{-m-3 l / 2} \lambda\left|n-n^{\prime}\right|
$$

if $\left|n-n^{\prime}\right|$ is large; this is more than enough to prove the required almost orthogonality property.

Now applying Proposition 6.1 to the functions

$$
F_{a \nu}^{m \mu b \tilde{b}}(y, \tilde{y})=\mathcal{L} f_{a \nu}^{m \mu b}(y) \mathcal{L} \tilde{f}_{a \nu}^{m \mu \tilde{b}}(\tilde{y})
$$

we reduced matters to

Proposition 6.4. For $2<r<\infty, r=3 p^{\prime} / 2$,

$$
\begin{aligned}
& \left(\sum_{0<m<l / 2} \sum_{\mu} \sum_{(b, \tilde{b}) \in \mathcal{P} m} 2^{-(m+3 l / 2) \frac{r^{\prime}}{r}}\left(\sum_{a, \nu}\left(\iint\left|\mathcal{L} f_{a \nu}^{m \mu b}(y) \mathcal{L} \tilde{f}_{a \nu}^{m \mu \tilde{b}}(\tilde{y})\right|^{r^{\prime}} d y d \tilde{y}\right)^{\frac{r}{r^{\prime}}}\right)^{\frac{r^{\prime}}{r}}\right)^{\frac{1}{r^{\prime}}} \\
& \lesssim 2^{-2 l / p^{\prime}}\|f\|_{p}\|\tilde{f}\|_{p} .
\end{aligned}
$$

For $r=2, p=4$ the left hand side of (6.33) is dominated by

$$
C(1+l)^{1 / 2} 2^{-3 l / 2}\|f\|_{4}\|\tilde{f}\|_{4} .
$$

Proof. Each $\mathcal{L} f_{a \nu}^{m \mu b}$ is supported on a parallelogram of area $\lesssim 2^{-m-3 l / 2}$ and so by Hölder's inequality we can estimate for $r \geq 2$ the left hand side of (6.33) by

$$
\begin{aligned}
& \left(\sum_{m<l / 2} \sum_{\mu} \sum_{(b, \tilde{b}) \in \mathcal{P}_{\mu}^{m}} 2^{-(m+3 l / 2) \frac{r^{\prime}}{r}} 2^{-(m+3 l / 2)\left(2-\frac{2 r^{\prime}}{p}\right)}\left(\sum_{a, \nu}\left\|\mathcal{L} f_{a \nu}^{m \mu b}\right\|_{p}^{r}\left\|\mathcal{L} \tilde{f}_{a \nu}^{m \mu \tilde{b}}\right\|_{p}^{r}\right)^{\frac{r^{\prime}}{r}}\right)^{\frac{1}{r^{\prime}}} \\
& \lesssim\left(\sum_{m<l / 2} 2^{-(m+3 l / 2)\left(\frac{r^{\prime}}{r}+2-\frac{2 r^{\prime}}{p}\right)} \sum_{(b, \tilde{b}) \in \mathcal{P}^{m}}\left\|f^{m b}\right\|_{p}^{r^{\prime}}\left\|\tilde{f}^{m \tilde{b}}\right\|_{p}^{r^{\prime}}\right)^{\frac{1}{r^{\prime}}}
\end{aligned}
$$

where $f^{m b}$ is an in (6.20). For the last inequality we have used the Cauchy-Schwarz inequality, the fact that $2 r \geq p$ (which follows from our assumptions on $p$ and $r$ ), the embedding $\ell^{p} \subset \ell^{2 r}$ and (6.21) of Lemma 6.3. 
Now let $I_{n}=\left[n 2^{-l / 2},(n+1) 2^{-l / 2}\right)$ for $n \in \mathbb{Z}$; then $I_{n}$ contains $\approx 2^{m}$ numbers $b \in$ $2^{-m-l / 2} \mathbb{Z}$ and we dominate (6.34) by a constant times

$$
\begin{aligned}
& \left(\sum_{m<l / 2} \sum_{\substack{(n, \tilde{n}) \in \mathbb{Z}^{2} \\
|n-\tilde{n}| \approx 2^{m}}} 2^{-(m+3 l / 2)\left(\frac{r^{\prime}}{r}+2-\frac{2 r^{\prime}}{p}\right)} 2^{2 m\left(1-\frac{r^{\prime}}{p}\right)}\right. \\
& \left.\quad \times\left(\sum_{\substack{b \in I_{n} \cap \\
2^{-m-l / 2} \mathbb{Z}}}\left\|f^{m b}\right\|_{p}^{p}\right)^{\frac{r^{\prime}}{p}}\left(\sum_{\substack{\tilde{b} \in I_{\tilde{n}} \cap \\
2^{-m-l / 2} \mathbb{Z}}}\left\|\tilde{f}^{m \tilde{b}}\right\|_{p}^{p}\right)^{\frac{r^{\prime}}{p}}\right)^{\frac{1}{r^{\prime}}} \\
& \lesssim 2^{-2 l / p^{\prime}}\left(\sum_{m} \sum_{\substack{(n, \tilde{n}): \\
|n-\tilde{n}| \approx 2^{m}}}|n-\tilde{n}|^{-r^{\prime}+1}\|f\|_{L^{p}\left(I_{n} \times \mathbb{R}\right)}^{r^{\prime}}\|\tilde{f}\|_{L^{p}\left(I_{\tilde{n}} \times \mathbb{R}\right)}^{r^{\prime}}\right)^{\frac{1}{r^{\prime}}} ;
\end{aligned}
$$

here we have used that $1 / r+2 / r^{\prime}-2 / p=4 /\left(3 p^{\prime}\right)$ in view of the assumption $r=q / 2=3 p^{\prime} / 2$.

Let $\beta \in(0,1)$ and define for a sequence $\mathfrak{a}$ the discrete analogue of the standard fractional integral

$$
\left[I^{\beta} \mathfrak{a}\right]_{n}=\sum_{\tilde{n}}|n-\tilde{n}|^{\beta-1} \mathfrak{a}_{\tilde{n}}
$$

Now the condition $r=3 p^{\prime} / 2$, is equivalent with $2-r^{\prime}=\frac{1}{p / r^{\prime}}-\frac{1}{\left(p / r^{\prime}\right)^{\prime}}$ so that for $2<r<\infty$, $r=3 p^{\prime} / 2$ the operator $I^{2-r^{\prime}}$ maps $\ell^{p / r^{\prime}} \rightarrow \ell^{\left(p / r^{\prime}\right)^{\prime}}$. We apply this with $\mathfrak{a}_{n}=\|f\|_{L^{p}\left(I_{n} \times \mathbb{R}\right)}^{r^{\prime}}$ and also set $\tilde{\mathfrak{a}}_{n}=\|\tilde{f}\|_{L^{p}\left(I_{n} \times \mathbb{R}\right)}^{r^{\prime}}$. Then the expression (6.36) is bounded by

$$
C 2^{-2 l / p^{\prime}}\left(\sum_{n} \tilde{\mathfrak{a}}_{n}\left[I^{2-r^{\prime}} \mathfrak{a}\right]_{n}\right)^{1 / r^{\prime}}
$$

and we argue as in Hörmander [24] to get

$$
\left(\sum_{n}\left|\tilde{\mathfrak{a}}_{n}\left[I^{2-r^{\prime}} \mathfrak{a}\right]_{n}\right|\right)^{1 / r^{\prime}} \leq\|\tilde{\mathfrak{a}}\|_{p / r^{\prime}}^{1 / r^{\prime}}\left\|I^{2-r^{\prime}} \mathfrak{a}\right\|_{\left(p / r^{\prime}\right)^{\prime}}^{1 / r^{\prime}} \lesssim\|\mathfrak{a}\|_{p / r^{\prime}}^{1 / r^{\prime}}\|\tilde{\mathfrak{a}}\|_{p / r^{\prime}}^{1 / r^{\prime}} \lesssim\|f\|_{p}\|\tilde{f}\|_{p} .
$$

The case $r=2, p=4$ is similar, except that the expression (6.36) is now estimated using a simple convolution inequality for each fixed $m$ and the sum over $m$ introduces the logarithmic term.

\section{Proof of Proposition 6.1}

We prove inequality (6.13) by interpolation between the extreme cases $r=2$ and $r=\infty$. The case $r=\infty$ is

$$
\begin{aligned}
\left\|\sum_{0<m<l / 2} \sum_{\mu \in 2^{-l / 2+m}} \sum_{\mathbb{Z} b, \tilde{b} \in \mathcal{P}_{\mu}^{m}} \sum_{a, \nu} \mathfrak{S}_{a \nu}^{m \mu b \tilde{b}} F_{a \nu}^{m \mu b \tilde{b}}\right\|_{\infty} \lesssim & \\
& \sum_{0<m<l / 2} \sum_{\mu} \sum_{(b, \tilde{b}) \in \mathcal{P}_{\mu}^{m}} \sup _{a, \nu} \iint\left|F_{a \nu}^{m \mu b \tilde{b}}(y, \tilde{y})\right| d y d \tilde{y} .
\end{aligned}
$$

This is immediate; one uses for fixed $m, \mu$ the almost disjointness of the cutoff functions $u_{a \nu}^{m \mu}$ in (6.7). 
For the remainder of this section we consider the case $r=2$ which is

$$
\begin{aligned}
\left\|\sum_{0<m<l / 2} \sum_{\mu \in 2^{-l / 2+m}} \sum_{\mathbb{Z}} \sum_{(b, \tilde{b}) \in \mathcal{P}_{\mu}^{m}} \sum_{a, \nu} \mathfrak{S}_{a \nu}^{m \mu b \tilde{b}} F_{a \nu}^{m \mu b \tilde{b}}\right\|_{2} \lesssim \\
\frac{1}{\lambda}\left(\sum_{0<m<l / 2} 2^{-(m+3 l / 2)} \sum_{\mu} \sum_{(b, \tilde{b}) \in \mathcal{P}_{\mu}^{m}} \sum_{a, \nu} \iint\left|F_{a \nu}^{m \mu b \tilde{b}}(y, \tilde{y})\right|^{2} d y d \tilde{y}\right)^{\frac{1}{2}} .
\end{aligned}
$$

7.1. The four steps in the proof. We need to use various orthogonality lemmata.

Lemma 7.1.1. For each $N \in \mathbb{N}$

$$
\begin{aligned}
& \left\|\sum_{0<m<l / 2} \sum_{\mu \in 2^{-l / 2+m}} \sum_{\mathbb{Z}(b, \tilde{b}) \in \mathcal{P}_{\mu}^{m}} \sum_{a, \nu} \mathfrak{S}_{a \nu}^{m \mu b \tilde{b}} F_{a \nu}^{m \mu b \tilde{b}}\right\|_{2} \lesssim
\end{aligned}
$$

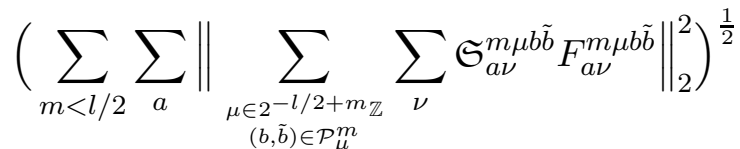

$$
\begin{aligned}
& +2^{-5 l / 4}\left(\lambda 2^{-2 l}\right)^{-N}\left(\sum_{m, \mu, b, \tilde{b}, a, \nu} 2^{-m(2 N-1)}\left\|F_{a \nu}^{m \mu b \tilde{b}}\right\|_{2}^{2}\right)^{\frac{1}{2}} .
\end{aligned}
$$

Lemma 7.1.2. For each $N \in \mathbb{N}$

$$
\begin{aligned}
& \text { (7.3) }\left\|\sum_{\mu \in 2^{-l / 2+m} \mathbb{Z}} \sum_{(b, \tilde{b}) \in \mathcal{P}_{\mu}^{m}} \sum_{\nu} \mathfrak{S}_{a \nu}^{m \mu b \tilde{b}} F_{a \nu}^{m \mu b \tilde{b}}\right\|_{2} \lesssim \\
& \left(\sum_{\mu \in 2^{-l / 2+m}} \sum_{\nu}\left\|\sum_{(b, \tilde{b}) \in \mathcal{P}_{\mu}^{m}} \mathfrak{S}_{a \nu}^{m \mu b \tilde{b}} F_{a \nu}^{m \mu b \tilde{b}}\right\|_{2}^{2}\right)^{1 / 2}+2^{-3 l / 2}\left(\lambda 2^{-2 l+m}\right)^{-N}\left(\sum_{\mu, b \tilde{b}, \nu}\left\|F_{a \nu}^{m \mu b \tilde{b}}\right\|_{2}^{2}\right)^{1 / 2},
\end{aligned}
$$

uniformly in $m$ and $a$.

Lemma 7.1.3. For each $N \in \mathbb{N}$

$$
\begin{aligned}
& \left\|\sum_{(b, \tilde{b}) \in \mathcal{P}_{\mu}^{m}} \mathfrak{S}_{a \nu}^{m \mu b \tilde{b}} F_{a \nu}^{m \mu b \tilde{b}}\right\|_{2} \lesssim \\
& \quad\left(\sum_{(b, \tilde{b}) \in \mathcal{P}_{\mu}^{m}}\left\|\mathfrak{S}_{a \nu}^{m \mu b \tilde{b}} F_{a \nu}^{m \mu b \tilde{b}}\right\|_{2}^{2}\right)^{1 / 2}+2^{-3(2 m+3 l) / 4}\left(\lambda 2^{-m-3 l / 2}\right)^{-N}\left(\sum_{b, \tilde{b}}\left\|F_{a \nu}^{m \mu b \tilde{b}}\right\|_{2}^{2}\right)^{1 / 2},
\end{aligned}
$$

uniformly in $m>0, \mu, a$ and $\nu$.

In view of our condition $2^{l} \leq \lambda^{1 / 3}$ the precise error bounds in the above lemmata will be unimportant.

These three estimates reduce matters to the uniform $L^{2}$ bounds for the operators $\mathfrak{S}_{a \nu}^{m \mu b \tilde{b}}$ :

Proposition 7.1.4. The estimate

$$
\left\|\mathfrak{S}_{a \nu}^{m \mu b \tilde{b}} F\right\|_{2} \lesssim 2^{-3 l / 4-m / 2} \lambda^{-1}\|F\|_{2}
$$

holds with bounds uniform in $m>0, \mu, a, \nu$ and $(b, \tilde{b}) \in \mathcal{P}_{\mu}^{m}$.

Inequality (6.13) for $r=2$ is an immediate consequence of Lemmata 7.1.1, 7.1.2, 7.1.3 and Proposition 7.1.4 we take into account that $2^{2 m} \leq 2^{l}$ and $2^{l} \leq \lambda^{1 / 3}$. 
7.2. Preliminary considerations. We first state a more or less standard result on oscillatory integrals, for which we include a sketch of the proof for completeness.

Lemma 7.2.1. Let $(x, y) \mapsto \Psi(x, y)$ be a smooth real valued phase function, defined in a domain $D \subset \mathbb{R}^{d} \times \mathbb{R}^{d}$ so that rank $\left(\Psi_{x^{\prime} y^{\prime}}\right)=d-1$ in $D$ and so that we have uniform bounds for the derivatives of $\Psi$ in $D$; i.e. ,

$$
\left|\partial_{x, y}^{\alpha} \Psi\right| \leq C_{\alpha}
$$

for all $|\alpha| \leq 4 d$. Let $\lambda \gg 1$ and $\delta \geq \lambda^{-1 / 3}$. Let $P^{o}=\left(x^{o}, y^{o}\right)$ and $Q_{\delta}\left(P^{o}\right)=\{(x, y)$ : $\left|x-x^{o}\right| \leq \delta,\left|y-y^{o}\right| \leq \delta$. $\}$. Suppose that for some $C_{1}>0$

$$
C_{1}^{-1} \delta \leq\left|\operatorname{det} \Psi_{x y}\right| \leq C_{1} \delta \text { for }(x, y) \in Q_{\delta}(P) .
$$

Let a be supported in $Q_{\delta}\left(P^{o}\right)$ and assume that

$$
\left|\partial_{x, y}^{\alpha} a\right| \leq C_{\alpha}(\lambda \delta)^{|\alpha| / 2}
$$

for all multiindices $\alpha$. Define the operator $\mathcal{J}_{\lambda}$ by

$$
\mathcal{J}_{\lambda} f(x)=\int e^{i \lambda \Psi(x, y)} a(x, y) f(y) d y .
$$

Then for $\lambda>\delta^{-3}$

$$
\left\|\mathcal{J}_{\lambda}\right\|_{L^{2} \rightarrow L^{2}} \lesssim \delta^{-1 / 2} \lambda^{-d / 2}
$$

where the implicit constants depend on $C_{1}$ in (7.7) and of a finite number of the constants in (7.6) $(|\alpha| \leq 10 d$ suffices).

Proof. We let $\delta_{1}=M^{-1} \delta$ where $M$ is very large in comparison to the constants in the assumptions (but independent of $\delta$ and $\lambda$ ). By a partition of unity we may assume that the symbol $a$ is supported in the smaller cube $Q \equiv Q_{\delta_{1}}\left(P^{o}\right)$.

We make affine changes of variables in $x$ and $y$ separately which do not affect the assumptions, so that we may assume that $P^{o}=O, \Psi_{x^{\prime} y^{\prime}}(O)=I_{d-1}($ the $(d-1) \times(d-1)$ identity matrix), and also $\Psi_{x^{\prime} y_{d}}(O)=0, \Psi_{y^{\prime} x_{d}}(O)=0$.

Then $\operatorname{det}\left(\Psi_{x y}\right)=\Psi_{x_{d} y_{d}}+O\left(\delta_{1}\right)$ in $Q$ and thus $\left|\Psi_{x_{d} y_{d}}\right| \approx\left|\operatorname{det}\left(\Psi_{x y}\right)\right| \approx \delta$. We shall use orthogonality arguments based on the following inequalities, valid for $(x, y) \in Q$ and $(x, z)$ in $Q$ :

$$
\left|\Psi_{x^{\prime}}(x, y)-\Psi_{x^{\prime}}(x, z)\right| \geq\left|y^{\prime}-z^{\prime}\right| \quad \text { if }\left|y^{\prime}-z^{\prime}\right| \geq C_{0} \delta_{1}\left|y_{d}-z_{d}\right|
$$

for a large constant $C_{0}$ and

$$
\left|\Psi_{x_{d}}(x, y)-\Psi_{x_{d}}(x, z)\right| \geq C^{-1} \delta\left|y_{d}-z_{d}\right| \quad \text { if }\left|y^{\prime}-z^{\prime}\right| \leq c_{0} \delta\left|y_{d}-z_{d}\right|
$$

for a small constant $c_{0}$ but $\delta_{1}$ is so small that $c_{0} \delta \gg C_{0} \delta_{1}$. Similar bounds hold for the phase $\Psi^{*}(x, y):=\Psi(y, x)$. Inequality (7.9) follows by a straightforward expansion about the origin, and it is crucial that we use $\Psi_{x^{\prime} y_{d}}(O)=0$. For (7.10) we use of course the lower bound on $\Psi_{x_{d} y_{d}}$.

We now decompose the amplitude into functions supported on rectangles $R_{m} \times R_{n}$ (with $\left.(m, n) \in \mathbb{Z}^{d} \times \mathbb{Z}^{d}\right)$ where both $R_{m}$ and $R_{n}$ have dimensions about $\lambda^{-1 / 2} \times \cdots \times \lambda^{-1 / 2} \times$ $\lambda^{-1 / 2} \delta^{-1 / 2}$. Let $\chi \in C_{0}^{\infty}(\mathbb{R})$ so that $\chi$ is supported in $(-5 / 4,5 / 4)$ and $\sum_{j \in \mathbb{Z}} \chi(s-j)=1$ 
for all $s \in \mathbb{R}$. Define for $(m, n) \in \mathbb{Z}^{d} \times \mathbb{Z}^{d}$

$$
\begin{aligned}
& a_{m, n}(x, y)= \\
& \left.\quad a(x, y) \chi\left(\lambda^{1 / 2} \delta^{1 / 2} x_{d}-m_{d}\right) \chi\left(\lambda^{1 / 2} \delta^{1 / 2} y_{d}-n_{d}\right) \prod_{i=1}^{d-1} \chi\left(\lambda^{1 / 2} x_{i}-m_{i}\right)\right) \prod_{i=1}^{d-1} \chi\left(\lambda^{1 / 2} y_{i}-n_{i}\right)
\end{aligned}
$$

and let $T_{m n}$ be defined as $\mathcal{J}_{\lambda}$ but with $a$ replaced by $a_{m n}$. Then $\mathcal{J}_{\lambda}=\sum_{m, n} T_{m n}$. We observe by simply using the support properties of the symbol and Schur's lemma that

$$
\left\|T_{m n}\right\|_{2-2} \lesssim \lambda^{-d / 2} \delta^{-1 / 2}
$$

moreover by disjointness of symbols

$$
\begin{array}{ll}
T_{p q}^{*} T_{m n}=0 & \text { if }|p-m| \geq 4, \\
T_{p q} T_{m n}^{*}=0 & \text { if }|q-n| \geq 4 .
\end{array}
$$

In order to use the Cotlar-Stein orthogonality lemma it suffices to show that

$$
\begin{aligned}
& \left\|T_{p q}^{*} T_{m n}\right\|_{2-2} \lesssim \lambda^{-d} \delta^{-1}\left|n-n^{\prime}\right|^{-N}, \text { for }|m-p| \leq C \text { and }|n-q| \geq C, \\
& \left\|T_{p q} T_{m n}^{*}\right\|_{2-2} \lesssim \lambda^{-d} \delta^{-1}|m-p|^{-N}, \text { for }|n-q| \leq C \text { and }|m-p| \geq C .
\end{aligned}
$$

Let $H_{m n p q}(y, z)$ be the Schwartz kernel of $T_{p q}^{*} T_{m n}$. By integration by parts we obtain the pointwise bounds

$$
\left|H_{m n p q}(y, z)\right| \lesssim \lambda^{-d / 2} \delta^{-1 / 2}\left(\frac{\frac{\left|y_{d}-z_{d}\right|}{\left|y^{\prime}-z^{\prime}\right|}+\sqrt{\lambda \delta}}{\lambda\left|y^{\prime}-z^{\prime}\right|}\right)^{N} \quad \text { if }\left|y^{\prime}-z^{\prime}\right| \geq C_{0} \delta_{1}\left|y_{d}-z_{d}\right|,
$$

and

$$
\left|H_{m n p q}(y, z)\right| \lesssim \lambda^{-d / 2} \delta^{-1 / 2}\left(\frac{\delta^{-2}+\sqrt{\lambda \delta}}{\lambda\left|y_{d}-z_{d}\right|}\right)^{N} \quad \text { if }\left|y^{\prime}-z^{\prime}\right| \leq c_{0} \delta\left|y_{d}-z_{d}\right| .
$$

and since $C_{0} \delta_{1} \ll c_{o} \delta$ all relevant situations are covered. In the first case we have $\left|y^{\prime}-z^{\prime}\right| \approx$ $\lambda^{-1 / 2}\left|m^{\prime}-p^{\prime}\right|$ and $\left|m^{\prime}-p^{\prime}\right| \gtrsim\left|m_{d}-p_{d}\right|$, and in the second case we have $\left|y_{d}-z_{d}\right| \approx$ $\lambda^{-1 / 2} \delta^{-1 / 2}\left|m_{d}-p_{d}\right|$ and $\left|m^{\prime}-p^{\prime}\right| \lesssim\left|m_{d}-p_{d}\right|$. By taking the support properties in $y$ and $z$ into account we can use Schur's Lemma to see that

$$
\begin{aligned}
& \sup _{y} \int\left|H_{m n p q}(y, z)\right| d z+\sup _{z} \int\left|H_{m n p q}(y, z)\right| d y \\
& \lesssim \lambda^{-d} \delta^{-1}\left\{\begin{array}{l}
\left(\frac{\delta^{-1}+\lambda^{1 / 2} \delta^{1 / 2}}{\lambda^{1 / 2}\left|m^{\prime}-p^{\prime}\right|}\right)^{N} \text { if }\left|m^{\prime}-p^{\prime}\right| \geq c\left|m_{d}-p_{d}\right|, \\
\left(\frac{\delta^{-3 / 2}+\lambda^{1 / 2} \delta}{\lambda^{1 / 2}\left|m_{d}-p_{d}\right|}\right)^{N} \text { if }\left|m^{\prime}-p^{\prime}\right| \leq C\left|m_{d}-p_{d}\right| .
\end{array}\right.
\end{aligned}
$$

Our restriction $\delta \geq \lambda^{-1 / 3}$ implies the desired bound (17.13) for the operator norm of $T_{p q}^{*} T_{m n}$. The operators $T_{p q} T_{m n}^{*}$ are handled analogously.

We now gather some facts that are useful for $L^{4}$ estimates related to the Carleson-Sjölin theorem. Define

$$
\begin{aligned}
& U_{1} \equiv U_{1}(s, \tilde{s}, t, \tilde{t})=s-t+\tilde{s}-\tilde{t} \\
& U_{2} \equiv U_{2}(s, \tilde{s}, t, \tilde{t})=(s-t)^{2}+(\tilde{s}-\tilde{t})^{2}-2(t-\tilde{t})(\tilde{s}-\tilde{t})
\end{aligned}
$$

and

$$
\widetilde{U}_{i}(s, \tilde{s}, t, \tilde{t})=U_{i}(\tilde{s}, s, \tilde{t}, t)
$$


moreover

$$
\begin{aligned}
& V_{i}(s, \tilde{s}, t, \tilde{t})=U_{i}(t, \tilde{t}, s, \tilde{s}) \\
& \widetilde{V}_{i}(s, \tilde{s}, t, \tilde{t})=\widetilde{U}_{i}(t, \tilde{t}, s, \tilde{s})
\end{aligned}
$$

(observe that $U_{1}=\widetilde{U}_{1}=-V_{1}=-\widetilde{V}_{1}$ ). The following calculus lemma is directly taken from p. 63 in [29]:

Lemma 7.2.2. Let $A=\left(A_{1}, A_{2}\right)$ be an $\mathbb{R}^{2}$-valued function of class $C^{4}$, defined on an interval. Suppose that $M, M^{\prime}>0$ and that $2^{-M-1} \leq s-\tilde{s} \leq 2^{-M+1}, 2^{-M^{\prime}-1} \leq t-\tilde{t} \leq$ $2^{-M^{\prime}+1}$. Let

$$
B(s, \tilde{s}, t, \tilde{t})=A(s)+A(\tilde{s})-A(t)-A(\tilde{t}) .
$$

Then (i)

$$
|B(s, \tilde{s}, t, \tilde{t})| \leq C \min \left\{\left|U_{1}\right|+\left|U_{2}\right|,\left|V_{1}\right|+\left|V_{2}\right|\right\}
$$

(ii) If also

$$
\left|A_{1}^{\prime}(s) A_{2}^{\prime \prime}(s)-A_{2}^{\prime}(s) A_{1}^{\prime \prime}(s)\right| \geq c_{1}
$$

then there is a uniform lower bound

$$
|B(s, \tilde{s}, t, \tilde{t})| \geq c \max \left\{\left|U_{1}\right|+\left|U_{2}\right|,\left|V_{1}\right|+\left|V_{2}\right|\right\} \quad \text { if }\left|M-M^{\prime}\right|>10 .
$$

(iii) There are constants $c>0, C_{1}>1$ so that if $M=M^{\prime}$ then the estimate

$$
|B(s, \tilde{s}, t, \tilde{t})| \geq c \max \left\{\left|U_{1}\right|+\left|U_{2}\right|,\left|V_{1}\right|+\left|V_{2}\right|\right\}
$$

holds in each of the following cases:

$$
\begin{aligned}
|s-t| & +|\tilde{s}-\tilde{t}| \leq C_{1}^{-1} 2^{-M} \\
\text { or }|s-t| & \geq C_{1} 2^{-M} \\
\text { or }|\tilde{s}-\tilde{t}| & \geq C_{1} 2^{-M} .
\end{aligned}
$$

(iv) There is a constant $C_{2}>1$ so that the following holds. Suppose that either $M<$ $M^{\prime}-20$ or $M=M^{\prime}$ and $|s-t|+|\tilde{s}-\tilde{t}| \geq C_{2} 2^{-M}$. Suppose that in addition $\left|U_{1}(s, \tilde{s}, t, \tilde{t})\right| \leq$ $2^{-M-10}$. Then

$$
\left|U_{2}(s, \tilde{s}, t, \tilde{t})\right| \geq \frac{1}{2}(\tilde{s}-\tilde{t})^{2} \geq 2^{-2 M-20} .
$$

(v) Suppose $M=M^{\prime}$ and let $\delta \leq 2^{-M-4}$. Suppose that $\left|U_{1}(s, \tilde{s}, t, \tilde{t})\right| \leq \delta / 4$ and suppose that $|s-t|+|\tilde{s}-\tilde{t}| \geq \delta$. Then $|s-t| \approx|\tilde{s}-\tilde{t}|$ and

$$
\left|U_{2}(s, \tilde{s}, t, \tilde{t})\right| \geq 2^{-M-1}|\tilde{s}-\tilde{t}| \geq c 2^{-M} \delta
$$

7.3. Proof of the orthogonality lemmata. For the proofs of Lemmata [7.1.1] 7.1.2 and 7.1.3, we shall need to analyze the expression

$$
\left\langle\mathfrak{S}_{a \nu}^{m \mu \nu \tilde{b}} F_{a \nu}^{m \mu \nu \tilde{b}}, \mathfrak{S}_{a^{\prime} \nu^{\prime}}^{m^{\prime} b^{\prime} \tilde{b}^{\prime}} F_{a^{\prime} \nu^{\prime}}^{m^{\prime} \mu^{\prime} b^{\prime} \tilde{b}^{\prime}}\right\rangle
$$

for the three cases $\left|m-m^{\prime}\right| \geq 20$, then $m=m^{\prime}$ and $\left|\mu-\mu^{\prime}\right| \gg C$ and finally $m=m^{\prime}$, $\mu=\mu^{\prime}$ and $|b-\tilde{b}|+\left|b^{\prime}-\tilde{b}^{\prime}\right| \gg C 2^{-m-l / 2}$. We shall apply the lower bounds of Lemma 7.2 .2 (ii) (with $M=l / 2-m, M^{\prime}=l / 2-m^{\prime}$ ) to the functions

$$
y_{1} \mapsto A\left(y_{1}\right):=\phi_{x}\left(x, y_{1}, g\left(x, y_{1}\right)\right)
$$


and the upper bounds of Lemma 7.2 .2 to higher $x$ derivatives of $\phi$, evaluated at $y=$ $\left(y_{1}, g\left(x, y_{1}\right)\right)$. The crucial Carleson-Sjölin type condition (7.22) holds, as by a straightforward calculation using (2.4) and (2.7)

$$
A_{1}^{\prime}\left(y_{1}\right) A_{2}^{\prime \prime}\left(y_{1}\right)-A_{2}^{\prime}\left(y_{1}\right) A_{1}^{\prime \prime}\left(y_{1}\right)=\left.\phi_{x_{1} y_{1}} \phi_{x_{2} y_{1} y_{1}}\right|_{\left(x, y_{1}, g\left(x, y_{1}\right)\right)}+o(1)
$$

where the $o(1)$ terms vanish at $O$ and the main terms are bounded below by the curvature condition (1.12) (in the reduced form (2.15)).

Now we use the notation $s=y_{1}, t=z_{1}, \tilde{s}=\tilde{y}_{1}, \tilde{t}=\tilde{z}_{1}$, and $U_{1} \equiv U_{1}\left(y_{1}, \tilde{y}_{1}, z_{1}, \tilde{z}_{1}\right)$ etc. Then we have

$$
\begin{array}{r}
\left|\partial_{x}^{\alpha} \phi\left(x, y_{1}, g\left(x, y_{1}\right)\right)+\partial_{x}^{\alpha} \phi\left(x, \tilde{y}_{1}, g\left(x, \tilde{y}_{1}\right)\right)-\partial_{x}^{\alpha} \phi\left(x, z_{1}, g\left(x, z_{1}\right)\right)-\partial_{x}^{\alpha} \phi\left(x, \tilde{z}_{1}, g\left(x, \tilde{z}_{1}\right)\right)\right| \\
\leq C_{\alpha} \min \left\{\left|U_{1}\right|+\left|U_{2}\right|,\left|V_{1}\right|+\left|V_{2}\right|\right\}
\end{array}
$$

and in the cases (i) $m^{\prime}<m-10$ and (ii) $m=m^{\prime}$ and one of (7.25), (77.26), (7.27) we also get the lower bounds

$$
\begin{aligned}
\left|\phi_{x}\left(x, y_{1}, g\left(x, y_{1}\right)\right)+\phi_{x}\left(x, \tilde{y}_{1}, g\left(x, \tilde{y}_{1}\right)\right)-\phi_{x}\left(x, z_{1}, g\left(x, z_{1}\right)\right)-\phi_{x}\left(x, \tilde{z}_{1}, g\left(x, \tilde{z}_{1}\right)\right)\right| \\
\geq c \max \left\{\left|U_{1}\right|+\left|U_{2}\right|,\left|V_{1}\right|+\left|V_{2}\right|\right\}
\end{aligned}
$$

In the four term expressions that occur in the phases when writing out (7.30) we have to replace $g\left(x, y_{1}\right)$ with $y_{2}$ etc. and then take into account that $(x, y)$ belongs to supp $\zeta_{l}$; this introduces error terms of size $O\left(2^{-l}\right)$.

Assuming that all points $(x, y),(x, z),(x, \tilde{y}),(x, \tilde{z})$ belong to the support of $\zeta_{l}$ then we obtain

$$
\left|\partial_{x}^{\alpha} \phi(x, y)+\partial_{x}^{\alpha} \phi(x, \tilde{y})-\partial_{x}^{\alpha} \phi(x, z)-\partial_{x}^{\alpha} \phi(x, \tilde{z})\right| \leq C_{\alpha}\left(\min \left\{\left|U_{1}\right|+\left|U_{2}\right|,\left|V_{1}\right|+\left|V_{2}\right|\right\}+2^{-l}\right) ;
$$

moreover in the cases described above we also get the lower bound

$$
\left|\phi_{x}(x, y)+\phi_{x}(x, \tilde{y})-\phi_{x}(x, z)-\phi_{x}(x, \tilde{z})\right| \geq c \max \left\{\left|U_{1}\right|+\left|U_{2}\right|,\left|V_{1}\right|+\left|V_{2}\right|\right\}-C 2^{-l} .
$$

In order to further bound below the right hand side of (7.32) we shall use the statements in part (iv) and (v) of Lemma 7.2.2. It will turn out that in all the described cases $\left|U_{1}\right|+\left|U_{2}\right| \gg 2^{-l}$ so that the error terms in (17.31) and (7.32) will not affect the integrations by parts. This is an important point of the proof, and many of our decompositions have been made with this goal in mind.

Finally, before we discuss the proofs of the lemmata we note that in all cases we may assume that $F_{a \nu}^{m \mu b \tilde{b}}$ is supported on a set of measure $2^{-2 l-3 m}$; simply replace $F_{a \nu}^{m \mu b \tilde{b}}$ with $\widetilde{\Omega}_{a \nu}^{m \mu b \tilde{b}} F_{a \nu}^{m \mu b \tilde{b}}$ where $\widetilde{\Omega}_{a \nu}^{m \mu b \tilde{b}} \Omega_{a \nu}^{m \mu b \tilde{b}}=\Omega_{a \nu}^{m \mu b \tilde{b}}$ and $\widetilde{\Omega}_{a \nu}^{m \mu b \tilde{b}}$ has support properties similar to $\Omega_{a \nu}^{m \mu b \tilde{b}}$. Thus

$$
\left\|F_{a \nu}^{m \mu b \tilde{b}}\right\|_{1} \lesssim 2^{-3 l / 2-m}\left\|F_{a \nu}^{m \mu b \tilde{b}}\right\|_{2} .
$$

Proof of Lemma 7.1.1. We square the right hand side of (7.2) and see that we need to analyze (7.30) with $\left|m-m^{\prime}\right| \geq 20$. By symmetry we may assume that $m^{\prime}<m-20$ (i.e. $\left.2^{-l / 2+m^{\prime}} \ll 2^{-l / 2+m}\right)$.

We also apply part (iv) of Lemma 7.2.2 which tells us that in the present situation $\left|U_{1}\right|+\left|U_{2}\right| \geq c 2^{-2 M} \equiv c 2^{-l+2 m}$. We integrate by parts and observe that if derivatives hit the symbols involved we get a factor of $2^{l}$ with each derivative. 
The size of the support of $u_{a \nu}^{m \mu}$ is $O\left(2^{-m-3 l / 2}\right)$. Consequently, after integrating by parts $2 N$ times, we obtain the bound

$$
\begin{aligned}
& \left|\left\langle\mathfrak{S}_{a \nu}^{m \mu b \tilde{b}} F_{a \nu}^{m \mu b \tilde{b}}, \mathfrak{S}_{a^{\prime} \nu^{\prime}}^{m^{\prime} \mu^{\prime} b^{\prime} \tilde{b}^{\prime}} F_{a^{\prime} \nu^{\prime}}^{m^{\prime} \mu^{\prime} b^{\prime} \tilde{b}^{\prime}}\right\rangle\right| \\
& \quad \leq C_{N}\left(\lambda 2^{-2 l+m}\right)^{-2 N} \operatorname{meas}\left(\operatorname{supp} u_{a \nu}^{m \mu} \cap u_{a^{\prime} \nu^{\prime}}^{m^{\prime} \mu^{\prime}}\right)\left\|F_{a \nu}^{m \mu b \tilde{b}}\right\|_{1}\left\|F_{a^{\prime} \nu^{\prime}}^{m^{\prime} \prime^{\prime} b^{\prime} \tilde{b}^{\prime}}\right\|_{1} \\
& \leq C_{N}^{\prime}\left(\lambda 2^{-2 l+m}\right)^{-2 N} 2^{-m-3 l / 2}\left\|F_{a \nu}^{m \mu b \tilde{b}}\right\|_{1}\left\|F_{a^{\prime} \nu^{\prime}}^{m^{\prime} \mu^{\prime} b^{\prime} \tilde{b}^{\prime}}\right\|_{1} .
\end{aligned}
$$

Now by the $T^{*} T$ argument using also the Cauchy-Schwarz inequality (for the terms with $\left.\left|m-m^{\prime}\right| \leq 20\right)$ the expression on the left hand side of (7.2) is dominated by $I+\sqrt{I I}$ where $I$ is the first term on the right hand side of (17.2) and

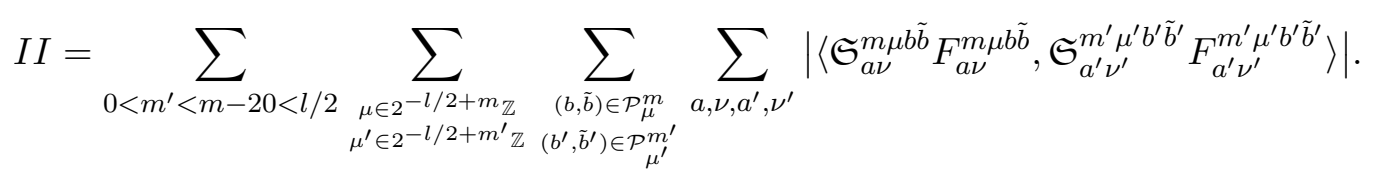

We also observe that for each fixed $m, m^{\prime}, \mu, \mu^{\prime}$ the sums in $(a, \nu)$ and $\left(a^{\prime}, \nu^{\prime}\right)$ are taken over index sets of cardinality $O\left(2^{3 l / 2+m}\right)$ and $O\left(2^{3 l / 2+m^{\prime}}\right)$, respectively. Moreover for each fixed $m, \mu$ the sums in $(b, \tilde{b})$ are over a set of cardinality $2^{4 m}$, and for each fixed $m^{\prime}, \mu^{\prime}$ the sums in $\left(b^{\prime}, \tilde{b}^{\prime}\right)$ are over a set of cardinality $2^{4 m^{\prime}}$. Finally for each fixed $m$ the sums in $\mu$ and $\mu^{\prime}$ are over sets of cardinalities $O\left(2^{l / 2-m}\right)$ and $O\left(2^{l / 2-m^{\prime}}\right)$, respectively. Taking these restrictions into account we continue with straightforward estimation using just the Cauchy-Schwarz inequality in the various parameters which gives an additional factor of $2^{3 l / 2+m / 2+m^{\prime} / 2} 2^{2 m+2 m^{\prime}} 2^{l / 2-m / 2-m^{\prime} / 2}$. We thus bound $|I I|$ by

$$
\begin{aligned}
& C_{N} \sum_{\substack{m, m^{\prime} \\
0<m^{\prime}<m-20}}\left(\lambda 2^{-2 l+m}\right)^{-2 N} 2^{-m-3 l / 2} \sum_{\substack{\mu \in 2^{-l / 2+m} \mathbb{Z} \\
\mu \in 2^{-l / 2+m^{\prime}} \mathbb{Z}}} \sum_{\substack{(b, \tilde{b}) \in \mathcal{P}_{\mu}^{m} \\
\left(b^{\prime}, \tilde{b}^{\prime}\right) \in \mathcal{P}_{\mu^{\prime}}^{m^{\prime}}}} \sum_{\substack{(a, \nu) \\
\left(a^{\prime}, \nu^{\prime}\right)}}\left\|F_{a \nu}^{m \mu \tilde{b}}\right\|_{1}\left\|F_{a^{\prime} \nu^{\prime}}^{m^{\prime} \mu^{\prime} b^{\prime} \tilde{b}^{\prime}}\right\|_{1} \\
& \leq C_{N}^{\prime} 2^{l / 2}\left(\lambda 2^{-2 l}\right)^{-2 N} \sum_{0<m<l / 2} 2^{(3-2 N) m} \sum_{\mu \in 2^{-l / 2+m} \mathbb{Z}} \sum_{\substack{(b, \tilde{b}) \in \mathcal{P}_{\mu}^{m} \\
(a, \nu)}} \sum_{(a \nu}\left\|F_{a \nu}^{m \mu \tilde{b}}\right\|_{1}^{2} .
\end{aligned}
$$

The assertion (17.2) follows if we choose $N$ large in the previous estimate and apply (7.33).

Proof of Lemma 7.1.2. Now $m$ is fixed and we need to bound (7.30) for $m=m^{\prime}$ and $\left|\mu-\mu^{\prime}\right| \geq C 2^{m-l / 2}$ for some large but absolute constant $C$. We argue as in the proof of Lemma 7.1.1, but now use Lemma 7.2.2 part (iii), (7.26) or (7.27), with $M=l / 2-m$. Thus the lower bound in (17.32) holds and also the upper bound in (17.31). For the lower bounds we have $\left|U_{1}\right|+\left|U_{2}\right| \geq c 2^{-2 M} \approx 2^{-l+2 m}$. Thus we get for $\left|\mu-\mu^{\prime}\right| \geq C 2^{m-l / 2}$,

$$
\left|\left\langle\mathfrak{S}_{a \nu}^{m \mu b \tilde{b}} F_{a \nu}^{m \mu b \tilde{b}}, \mathfrak{S}_{a^{\prime} \nu^{\prime}}^{m \mu^{\prime} b^{\prime} \tilde{b}^{\prime}} F_{a^{\prime} \nu^{\prime}}^{m \mu^{\prime} b^{\prime} \tilde{b}^{\prime}}\right\rangle\right| \leq C_{N}\left(\lambda 2^{-2 l+m}\right)^{-2 N} 2^{-m-3 l / 2}\left\|F_{a \nu}^{m \mu b \tilde{b}}\right\|_{1}\left\|F_{a^{\prime} \nu^{\prime}}^{m \mu^{\prime} b^{\prime} \tilde{b}^{\prime}}\right\|_{1} .
$$

From here we proceed as in the proof of Lemma 7.1.1 we use the Cauchy-Schwarz inequality in the parameters $\mu, \mu^{\prime},(b, \tilde{b}),\left(b^{\prime}, \tilde{b}^{\prime}\right)$, and $\nu$, and then (7.33).

Remark. One could also use Fourier transform arguments (with respect to $x$ ) as in the proof of Proposition 8.1 below.

Proof of Lemma 7.1.3. We have now $m, \mu, a$ and $\nu$ fixed, and we are required to estimate $\left\|\sum_{b, \tilde{b} \in \mathcal{P}_{\mu}^{m}} \mathfrak{S}_{a \nu}^{m \mu b \tilde{b}} F_{a \nu}^{m \mu b b}\right\|_{2}$. The relevant $(b, \tilde{b})$ is such that $|b-\mu| \leq C 2^{-l / 2+m}$ and $|\tilde{b}-\mu| \leq$ $C 2^{-l / 2+m}$. 
We split the family of pairs $\mathcal{P}_{\mu}^{m}$ into a bounded set of subfamilies $\mathcal{P}_{\mu, i}^{m}$ with the property that for any two pairs $(b, \tilde{b}),\left(b^{\prime}, \tilde{b}^{\prime}\right)$ in one such $\mathcal{P}_{\mu, i}^{m}$ we have both $\left|b-b^{\prime}\right| \leq c 2^{-l / 2+m}$ and $\left|\tilde{b}-\tilde{b}^{\prime}\right| \leq c 2^{-l / 2+m}$ for a small constant $c$.

This time we need to analyze (7.30) with $m=m^{\prime}, \mu=\mu^{\prime}$ and $(b, \tilde{b}) \in \mathcal{P}_{\mu, i}^{m},\left(b^{\prime}, \tilde{b}^{\prime}\right) \in \mathcal{P}_{\mu, i}^{m}$. We may use integration by parts since by the definition of $\mathcal{P}_{\mu, i}^{m}$ we are in the situation of part (iii), (7.25) of Lemma 7.2.2, with $M=l / 2-m$. The lower bound $\left|U_{1}\right|+\left|U_{2}\right| \geq 2^{-M} \delta$ in (7.29) applies with

$$
\delta \approx\left|b-b^{\prime}\right|+\left|\tilde{b}-\tilde{b}^{\prime}\right| \geq C_{4} 2^{-m-l / 2},
$$

for some large $C_{4}$. Thus in this case

$$
\begin{aligned}
& \left|\left\langle\mathfrak{S}_{a \nu}^{m \mu b \tilde{b}} F_{a \nu}^{m \mu b \tilde{b}}, \mathfrak{S}_{a^{\prime} \nu^{\prime}}^{m \mu b^{\prime} \tilde{b}^{\prime}} F_{a^{\prime} \nu^{\prime}}^{m \mu \prime^{\prime} \tilde{b}^{\prime}}\right\rangle\right| \\
& \quad \lesssim 2^{-m-3 l / 2}\left(\lambda 2^{-m-3 l / 2}\left(\left|b-b^{\prime}\right|+\left|\tilde{b}-\tilde{b}^{\prime}\right|\right)\right)^{-2 N}\left\|F_{a \nu}^{m \mu b \tilde{b}}\right\|_{1}\left\|F_{a^{\prime} \nu^{\prime}}^{m \mu b^{\prime} \tilde{b}^{\prime}}\right\|_{1}
\end{aligned}
$$

for $(b, \tilde{b}) \in \mathcal{P}_{\mu, i}^{m},\left(b^{\prime}, \tilde{b}^{\prime}\right) \in \mathcal{P}_{\mu, i}^{m}$ satisfying (7.35). By a straightforward convolution inequality

$$
\begin{aligned}
\| \sum_{(b, \tilde{b}) \in \mathcal{P}_{\mu}^{m}} & \mathfrak{S}_{a \nu}^{m \mu b \tilde{b}} F_{a \nu}^{m \mu b \tilde{b}} \|_{2} \lesssim \\
& \left(\sum_{(b, \tilde{b}) \in \mathcal{P}_{\mu}^{m}}\left\|\mathfrak{S}_{a \nu}^{m \mu b \tilde{b}} F_{a \nu}^{m \mu b \tilde{b}}\right\|_{2}^{2}\right)^{1 / 2}+2^{-m / 2-3 l / 4}\left(\lambda 2^{-m-3 l / 2}\right)^{-N}\left(\sum_{b, \tilde{b}}\left\|F_{a \nu}^{m \mu b \tilde{b}}\right\|_{1}^{2}\right)^{1 / 2},
\end{aligned}
$$

and (17.33) is used to obtain the desired conclusion.

7.4. Proof of Proposition [7.1.4. This is to be deduced from Lemma 7.2.1. We change variables in the integral defining $\mathfrak{S}_{a \nu}^{m \mu b \tilde{b}}$ to

$$
y_{2}=g\left(\tau_{\nu}^{\mu} a, y_{1}\right)+\sigma, \quad \tilde{y}_{2}=g\left(\tau_{\nu}^{\mu} a, y_{1}\right)+\tilde{\sigma},
$$

where then integrations over $\sigma, \tilde{\sigma}$ are extended over intervals of length $O\left(2^{-l}\right)$.

We then have

$$
\mathfrak{S}_{a \nu}^{m \mu b \tilde{b}} F(x)=\left(\eta\left(2^{l}\left(\left\langle\frac{\nabla_{x} g(a, \mu)}{\left|\nabla_{x} g(a, \mu)\right|}, x-\tau_{\nu}^{\mu} a\right\rangle\right)\right)\right)^{2} \iint_{|\sigma|,|\tilde{\sigma}| \lesssim 2^{-l}} \mathcal{T}_{a \nu, \sigma \tilde{\sigma}}^{m \mu b \tilde{b}}\left[H_{\sigma \tilde{\sigma}} F\right] d \sigma d \tilde{\sigma}
$$

where

$$
H_{\sigma \tilde{\sigma}} F\left(y_{1}, \tilde{y}_{1}\right)=\beta_{0}\left(C^{-1} 2^{l / 2} \sigma\right) \beta_{0}\left(C^{-1} 2^{l / 2} \tilde{\sigma}\right) F\left(y_{1}, g\left(\tau_{\nu}^{\mu} a, y_{1}\right)+\sigma, \tilde{y}_{1}, g\left(\tau_{\nu}^{\mu} a, \tilde{y}_{1}\right)+\tilde{\sigma}\right) .
$$

The oscillatory integral operators $\mathcal{T}_{a \nu, \sigma \tilde{\sigma}}^{m \mu \tilde{b}}$ in (7.36) act on functions $h$ of the variables $\left(y_{1}, \tilde{y}_{1}\right)$ and are defined by

$$
\mathcal{T}_{a \nu, \sigma \tilde{\sigma}}^{m \mu b \tilde{b}} h(x)=\iint \mathcal{A}\left(x, y_{1}, \tilde{y}_{1} ; \sigma, \tilde{\sigma}\right) e^{i \lambda \Psi\left(x, y_{1}, \tilde{y}_{1} ; \sigma, \tilde{\sigma}\right)} h\left(y_{1}, \tilde{y}_{1}\right) d y_{1} d \tilde{y}_{1}
$$

with

$$
\begin{aligned}
\mathcal{A}\left(x, y_{1}, \tilde{y}_{1} ; \sigma, \tilde{\sigma}\right)=\left(u_{a}^{m}(x)\right)^{2} \chi_{1}\left(2^{l / 2-m-5}\left(y_{1}-\tilde{y}_{1}\right)\right) \zeta_{l}\left(x, y_{1}, g\left(\tau_{\nu}^{\mu} a, y_{1}\right)+\sigma\right) \\
\times \zeta_{l}\left(x, \tilde{y}_{1}, g\left(\tau_{\nu}^{\mu} a, y_{1}\right)+\tilde{\sigma}\right) \Omega_{a}^{m b \tilde{b}}\left(y_{1}, g\left(\tau_{\nu}^{\mu} a, y_{1}\right)+\sigma, \tilde{y}_{1}, g\left(\tau_{\nu}^{\mu} a, y_{1}\right)+\tilde{\sigma}\right)
\end{aligned}
$$

and

$$
\Psi\left(x, y_{1}, \tilde{y}_{1} ; \sigma, \tilde{\sigma}\right)=\phi\left(x, y_{1}, g\left(\tau_{\nu}^{\mu} a, y_{1}\right)+\sigma\right)+\phi\left(x, \tilde{y}_{1}, g\left(\tau_{\nu}^{\mu} a, \tilde{y}_{1}\right)+\tilde{\sigma}\right)
$$


By the Cauchy-Schwarz inequality

$$
\left\|\mathfrak{S}_{a \nu}^{m \mu b \tilde{b}} F\right\|_{2} \lesssim 2^{-l}\left(\iint_{|\sigma|,|\tilde{\sigma}| \lesssim 2^{-l}}\left\|\mathcal{T}_{a \nu, \sigma \tilde{\sigma}}^{m \mu \tilde{b}}\left[H_{\sigma \tilde{\sigma}} F\right]\right\|_{2}^{2} d \sigma d \tilde{\sigma}\right)^{1 / 2}
$$

One now verifies that Lemma 7.2 .1 with $\delta \approx 2^{m-l / 2}$ can be applied to the operators $\mathcal{T}_{a \nu, \sigma \tilde{\sigma}}^{m \mu \tilde{b}}$ so that the right hand side of (7.40) is estimated by a constant times

$$
2^{-m / 2-3 l / 4}\left(\iint_{|\sigma|,|\tilde{\sigma}| \lesssim 2^{-l}}\left\|H_{\sigma \tilde{\sigma}} F\right\|_{2}^{2} d \sigma d \tilde{\sigma}\right)^{1 / 2} \lesssim 2^{-m / 2-3 l / 4}\|F\|_{2} .
$$

\section{Estimation of $\mathfrak{B}^{0}(f, f)$}

This case is handled rather analogously to the case $m>0$, except instead of using Proposition 7.1.4 we reduce directly to the Carleson-Sjölin theorem.

We shall set $\mathfrak{S}_{a, \nu}^{\mu b \tilde{b}}:=\mathfrak{S}_{a, \nu}^{0 \mu b \tilde{b}}, \mathcal{P}_{\mu}:=\mathcal{P}_{\mu}^{0}$, moreover $f_{a \nu}^{\mu b}(y):=\omega_{a \nu}^{0 \mu b}(y) f(y)(c f$. (6.12) ), and define the expressions $\mathcal{L} f_{a \nu}^{\mu b}(y)$ and $\mathcal{E} f_{a \nu}^{\mu b}(y)$ by setting $m=0$ in (6.15) and (6.16). (Note that $\mathcal{P}_{\mu}$ contains boundedly many elements for each $\mu$.)

Note that Lemma 6.2 remains valid for $m=0$ so that $\mathfrak{S}_{a, \nu}^{0 \mu b \tilde{b}}$ essentially acts on $\mathcal{L} f_{a \nu}^{\mu b} \otimes$ $\mathcal{L} f_{a \nu}^{\mu \tilde{b}}$. Various orthogonality arguments will be used for the proof of

Proposition 8.1. For $r \geq 2$ and $N \in \mathbb{N}$,

$$
\begin{aligned}
\| \sum_{\mu \in 2^{-l} / 2} & \sum_{\mathbb{Z}} \sum_{(b, \tilde{b}) \in \mathcal{P}_{\mu}} \mathfrak{S}_{a, \nu}^{0 \mu b \tilde{b}}\left(f_{a \nu}^{\mu b} \otimes f_{a \nu}^{\mu \tilde{b}}\right) \|_{r} \lesssim \\
& \left(\sum_{a, \nu} \sum_{\mu, b}\left\|\mathfrak{S}_{a, \nu}^{0 \mu b \tilde{b}}\left(\mathcal{L} f_{a \nu}^{\mu b} \otimes \mathcal{L} f_{a \nu}^{\mu \tilde{b}}\right)\right\|_{r}^{r^{\prime}}\right)^{1 / r^{\prime}}+C_{N} \lambda^{3}\left(\lambda 2^{-2 l}\right)^{-N / r} \sup _{a, \nu, \mu, b}\left\|f_{a \nu}^{\mu b}\right\|_{1}^{2} .
\end{aligned}
$$

We combine this with an application of the Carleson-Sjölin theorem which will give

Proposition 8.2. For $1 \leq p<4, r=3 p^{\prime} / 2$,

$$
\left\|\mathfrak{S}_{a, \nu}^{0 \mu b \tilde{b}}(f \otimes g)\right\|_{r} \lesssim 2^{-2 l / p^{\prime}} \lambda^{-4 /\left(3 p^{\prime}\right)}\|f\|_{p}\|g\|_{p}
$$

moreover

$$
\left\|\mathfrak{S}_{a, \nu}^{0 \mu b \tilde{b}}(f \otimes g)\right\|_{2} \lesssim 2^{-3 l / 2} \lambda^{-1}(\log \lambda)^{1 / 2}\|f\|_{4}\|g\|_{4} .
$$

The error term in (8.1) is easily bounded by the right hand side of (6.3) or (6.4) given that $2^{l} \lesssim \lambda^{1 / 3}$. For the main term in (8.1) we apply Proposition 8.2 with $f \otimes g=\left(\mathcal{L} f_{a \nu}^{\mu b} \otimes \mathcal{L} f_{a \nu}^{\mu \tilde{b}}\right)$ and put the result into (8.1); this yields

$$
\begin{aligned}
\left(\sum_{a, \nu} \sum_{\mu, b}\left\|\mathfrak{S}_{a, \nu}^{0 \mu b \tilde{b}}\left(\mathcal{L} f_{a \nu}^{\mu b} \otimes \mathcal{L} f_{a \nu}^{\mu \tilde{b}}\right)\right\|_{r}^{r^{\prime}}\right)^{1 / r^{\prime}} & \lesssim A_{p}(\lambda, l)\left(\sum_{a, \nu} \sum_{\mu, b}\left\|\mathcal{L} f_{a \nu}^{\mu b}\right\|_{p}^{2 r^{\prime}}\right)^{1 / r^{\prime}} \\
& \lesssim A_{p}(\lambda, l)\left(\sum_{a, \nu} \sum_{\mu, b}\left\|\mathcal{L} f_{a \nu}^{\mu b}\right\|_{p}^{p}\right)^{2 / p}
\end{aligned}
$$

where $A_{p}(\lambda, l)=2^{-2 l / p^{\prime}} \lambda^{-4 /\left(3 p^{\prime}\right)}$ if $p>4$ and $A_{4}(\lambda, l)=2^{-3 l / 2} \lambda^{-1}(\log \lambda)^{1 / 2}$. In the last displayed inequality we have used that if $r=3 p^{\prime} / 2$ then $2 r^{\prime} \geq p$ holds for $p \leq 4$. The desired estimate for $\mathfrak{B}_{0}(f, f)$ then follows from an application of Lemma 6.3 to (8.4). 
Proof of Proposition 8.1. The $u_{a}^{0}$ are supported on cubes $Q_{a}$ with diameter $\approx 2^{-l / 2}$, centered at $a$, which are essentially disjoint (so that $\sum_{a} \chi_{Q_{a}}(x) \leq C$ ). Thus

$$
\begin{aligned}
\left\|\sum_{\mu \in 2^{-l / 2} \mathbb{Z}} \sum_{(b, \tilde{b}) \in \mathcal{P}_{\mu}} \sum_{a, \nu} \mathfrak{S}_{a, \nu}^{0 \mu b \tilde{b}}\left(f_{a \nu}^{\mu b} \otimes f_{a \nu}^{\mu \tilde{b}}\right)\right\|_{r} \\
\qquad\left(\sum_{a}\left\|\chi_{Q_{a}} \sum_{\mu \in 2^{-l / 2} \mathbb{Z}} \sum_{(b, \tilde{b}) \in \mathcal{P}_{\mu}} \sum_{\nu} \mathfrak{S}_{a, \nu}^{0 \mu b \tilde{b}}\left(f_{a \nu}^{\mu b} \otimes f_{a \nu}^{\mu \tilde{b}}\right)\right\|_{r}^{r}\right)^{1 / r} .
\end{aligned}
$$

Let $\eta_{0} \in C_{0}^{\infty}\left(\mathbb{R}^{2}\right)$ be such that $\eta_{0}(s)=1$ if $|s| \leq 1$. Let $W_{a \mu}$ be the convolution operator on functions in $\mathbb{R}^{2}$ which has Fourier multiplier

$$
w_{a \mu}(\xi)=\eta_{0}\left(C^{-1} \lambda^{-1} 2^{l / 2}\left(\xi-2 \lambda \nabla_{x} \phi(a, \mu, g(a, \mu))\right)\right) ;
$$

here $C$ is chosen so large that $\left|\lambda^{-1} \xi-\nabla_{x} \phi(x, y)-\nabla_{x} \phi(x, \tilde{y})\right| \geq 2^{-l / 2}$ whenever $w_{a \mu}(\xi)=0$ and $(x, y, \tilde{y})$ is in the convex hull of the support of $u_{a}^{m}(x) \omega_{a}^{m b}\left(y_{1}\right) \omega_{a}^{m \tilde{b}}\left(\tilde{y}_{1}\right)$ for all $(b, \tilde{b}) \in \mathcal{P}_{\mu}$.

In view of this property we obtain by the inversion formula for the Fourier transform and a straightforward integration by parts argument that

$$
\begin{aligned}
\left|\left(I-W_{a \mu}\right) \mathfrak{S}_{a, \nu}^{0 \mu b \tilde{b}}\left(f_{a \nu}^{\mu b} \otimes f_{a \nu}^{\mu \tilde{b}}\right)(x)\right| & \leq\left\|\left(1-w_{a \mu}\right)\left(\mathfrak{S}_{a, \nu}^{0 \mu b \tilde{b}}\left(f_{a \nu}^{\mu b} \otimes f_{a \nu}^{\mu \tilde{b}}\right)\right)^{\wedge}\right\|_{1} \\
& \leq C_{N} \lambda^{2} 2^{-2 l}\left(\lambda 2^{-3 l / 2}\right)^{-N}\left\|f_{a \nu}^{\mu b}\right\|_{1}\left\|f_{a \nu}^{\mu \tilde{b}}\right\|_{1}
\end{aligned}
$$

for all $N \in \mathbb{N}$, uniformly in $x \in \mathbb{R}^{2}$. From this the contribution

$$
\left(\sum_{a}\left\|\chi_{Q_{a}} \sum_{\mu \in 2^{-l / 2} \mathbb{Z}} \sum_{(b, \tilde{b}) \in \mathcal{P}_{\mu}} \sum_{\nu}\left(I-W_{a \mu}\right) \mathfrak{S}_{a, \nu}^{0 \mu \tilde{b}}\left(f_{a \nu}^{\mu b} \otimes f_{a \nu}^{\mu \tilde{b}}\right)\right\|_{r}^{r}\right)^{1 / r}
$$

can be estimated by the error term in (8.1) in a straightforward way (we use that there are $O\left(2^{l}\right)$ relevant $a$ 's, $O\left(2^{l / 2}\right)$ relevant $\mu$ 's, and for fixed $a, \mu$ there are $O\left(2^{l / 2}\right)$ relevant $\mu$ 's and $O(1)$ relevant $b$ 's).

For the main term we use the orthogonality properties of the operators $W_{a \mu}$ (with respect to $\mu$ when $a$ is fixed) and then the essential disjoint support of the functions $u_{a, \nu}^{0 \mu}$ (when $a, \mu$ are fixed). We obtain

$$
\begin{aligned}
\left(\sum_{a}\left\|\chi_{Q_{a}} \sum_{\mu \in 2^{-l / 2} \mathbb{Z}} \sum_{(b, \tilde{b}) \in \mathcal{P}_{\mu}} \sum_{\nu} W_{a \mu} \mathfrak{S}_{a, \nu}^{0 \mu \nu \tilde{b}}\left(f_{a \nu}^{\mu b} \otimes f_{a \nu}^{\mu \tilde{b}}\right)\right\|_{r}^{r}\right)^{1 / r} & \\
& \lesssim\left(\sum_{a}\left(\sum_{\mu \in 2^{-l / 2} \mathbb{Z}} \sum_{(b, \tilde{b}) \in \mathcal{P}_{\mu}} \sum_{\nu}\left\|\mathfrak{S}_{a, \nu}^{0 \mu b \tilde{b}}\left(f_{a \nu}^{\mu b} \otimes f_{a \nu}^{\mu \tilde{b}}\right)\right\|_{r}^{r^{\prime}}\right)^{r / r^{\prime}}\right)^{1 / r}
\end{aligned}
$$

Finally using Lemma 6.2 we can replace $\left(f_{a \nu}^{\mu b} \otimes f_{a \nu}^{\mu \tilde{b}}\right)$ by $\left(\mathcal{L} f_{a \nu}^{\mu b} \otimes \mathcal{L} f_{a \nu}^{\mu \tilde{b}}\right)$ as the other terms just contribute to the error term in (8.1).

\section{Proof of Proposition 8.2. Define}

$$
T_{a \nu}^{\mu b} f(x)=u_{b \nu}^{0 \mu}(x) \int \zeta_{l}(x, y) \omega_{a \nu}^{0 \mu b}(y) e^{i \lambda \phi(x, y)} f(y) d y
$$

We dispose of the diagonal cutoff function $\chi_{0}\left(2^{l / 2-5}\left(y_{1}-\tilde{y}_{1}\right)\right)$ in the definition of $\mathfrak{S}_{a \nu}^{0 \mu b \tilde{b}}$ by expanding $\chi_{0}$ in a Fourier series and obtain

$$
\mathfrak{S}_{a \nu}^{0 \mu b \tilde{b}}(f \otimes g)=\sum_{k \in \mathbb{Z}} c_{k} T_{a \nu}^{\mu b} f_{k}(x) T_{a \nu}^{\mu b} g_{k}(x)
$$


where $f_{k}(y)=f(y) e^{i k 2^{l / 2-5} y_{1}}, g_{k}(y)=g(y) e^{-i k 2^{l / 2-5} y_{1}}$ and $\left|c_{k}\right| \leq C_{N} 2^{-N|k|}$ for all $N \in \mathbb{N}$. Then

$$
\left\|\mathfrak{S}_{a, \nu}^{0 \mu b \tilde{b}}(f \otimes g)\right\|_{r} \lesssim \sup _{k}\left\|T_{a \nu}^{\mu b} f_{k}\right\|_{2 r}\left\|T_{a \nu}^{\mu \tilde{b}} g_{k}\right\|_{2 r} .
$$

We now change variables $y_{2}=g(a, b)+\sigma$ in (8.7) and in view of the support assumption the $\sigma$ integration is extended over an interval of length $\leq C 2^{-l}$. The phase $\Psi^{\sigma}\left(x, y_{1}\right)=$ $\Phi\left(x, y_{1}, g(a, b)+\sigma\right)$ is a phase satisfying the assumptions of the Carleson-Sjölin theorem with bounds uniform in the parameters. Thus if we set

$$
T_{a \nu}^{\mu b, \sigma} h(x)=u_{b \nu}^{0 \mu}(x) \int \zeta_{l}\left(x, y_{1}, g(a, b)+\sigma\right) \omega_{a \nu}^{0 \mu b}\left(y_{1}, g(a, b)+\sigma\right) e^{i \lambda \phi\left(x, y_{1}, g(a, b)+\sigma\right)} h\left(y_{1}\right) d y_{1}
$$

we obtain with $2 r=3 p^{\prime}, p<4$

$$
\begin{aligned}
\left\|T_{a \nu}^{\mu b} f_{k}\right\|_{2 r} & \leq \int_{|\sigma| \leq C 2^{-l}}\left\|T_{a \nu}^{\mu b, \sigma}\left[f_{k}(\cdot, g(a, b)+\sigma)\right]\right\|_{2 r} d \sigma \\
& \lesssim \lambda^{-2 / 2 r} \int_{|\sigma| \leq C 2^{-l}}\left\|f_{k}(\cdot, g(a, b)+\sigma)\right\|_{p} d \sigma \\
& \lesssim 2^{-l / p^{\prime}} \lambda^{-3 / p^{\prime}}\left\|f_{k}\right\|_{p},
\end{aligned}
$$

and of course $\left\|f_{k}\right\|_{p}=\|f\|_{p}$. We argue similarly in the case $p=2 r=4$ but then one gets an additional factor $(\log \lambda)^{1 / 4}$ in the bound.

\section{Appendix I:}

A Sharpening of AN $L^{p}$ IMPROVING INEQUALITY FOR AVERAGES ON CURVES

Consider the translation invariant averaging operator

$$
\mathcal{A} f(x)=\int \chi(s) f(x-\gamma(s)) d s
$$

for the curve

$$
\gamma(s)=\left(s, \frac{s^{2}}{2}, \frac{s^{3}}{6}\right)
$$

where $\chi$ denotes a cutoff function to a neighborhood of 0 . The sharp $L^{p} \rightarrow L^{q}$ estimates are known and due to Oberlin [30, in fact $\mathcal{A}$ maps $L^{p} \rightarrow L^{q}$ if and only if $(1 / p, 1 / q)$ belongs to the trapezoid with corners $(0,0),(1,1),(1 / 2,1 / 3)$ and $(2 / 3,1 / 2)$. However the critical $L^{2} \rightarrow L^{3}$ and $L^{3 / 2} \rightarrow L^{2}$ estimates can be improved if one uses Lorentz spaces; this improvement does to the best of our knowledge not follow from the $T^{*} T$ method used in 30.

Theorem 9.1. $\mathcal{A}$ maps $L^{2}\left(\mathbb{R}^{3}\right)$ to $L^{3,2}\left(\mathbb{R}^{3}\right)$ and $L^{3 / 2,2}\left(\mathbb{R}^{3}\right)$ to $L^{2}\left(\mathbb{R}^{3}\right)$.

Proof. By duality it suffices to prove the $L^{2} \rightarrow L^{3,2}$ inequality. By a standard reduction using Littlewood-Paley theory it suffices to prove that the operators $\mathcal{A}_{k}$ defined by

$$
\mathcal{A}_{k} f(x)=\int f(y) \iint e^{i \sigma\left(x_{2}-y_{2}-\frac{\left(x_{1}-y_{1}\right)^{2}}{2}\right)+i \tau\left(x_{3}-y_{3}-\frac{\left(x_{1}-y_{1}\right)^{3}}{6}\right)} \chi_{1}\left(\frac{\sigma^{2}+\tau^{2}}{2^{2 k}}\right) \chi(x-y) d \sigma d \tau d y
$$

map $L^{2}$ to $L^{3,2}$ boundedly (with norms uniformly in $k \gg 0$ ). Here $\chi_{1} \in C_{0}^{\infty}(\mathbb{R})$ is an appropriate cutoff function supported away from 0 . The reason for the validity of this reduction is that $\mathcal{A}_{k}=L_{k} \mathcal{A}_{k} L_{k}+E_{k}$ where $L_{k}$ are Littlewood-Paley operators localizing 
frequencies to annuli of width $C 2^{k}$ and the errors $E_{k}$ satisfy $\left\|E_{k}\right\|_{L^{p} \rightarrow L^{q}}=O\left(2^{-k}\right)$. Then assuming that

$$
\sup _{k}\left\|\mathcal{A}_{k}\right\|_{L^{2} \rightarrow L^{3 / 2}} \leq A
$$

we obtain

$$
\begin{aligned}
& \left\|\sum_{k>0} L_{k} \mathcal{A}_{k} L_{k} f\right\|_{L^{3,2}} \lesssim\left\|\left(\sum_{k>0}\left|\mathcal{A}_{k} L_{k} f\right|^{2}\right)^{1 / 2}\right\|_{L^{3,2}} \\
& \lesssim\left(\sum_{k>0}\left\|\mathcal{A}_{k} L_{k} f\right\|_{L^{3,2}}^{2}\right)^{1 / 2} \lesssim A\left(\sum_{k>0}\left\|L_{k} f\right\|_{L^{2}}^{2}\right)^{1 / 2} \lesssim A\|f\|_{2}
\end{aligned}
$$

For the first inequality we used Littlewood-Paley theory, and for the second one we used a Minkowski-type inequality which amounts to the imbedding $\ell^{2}\left(L^{3 / 2}\right) \subset L^{3,2}\left(\ell^{2}\right)$ which can be seen using the equivalence $\left\|u^{2}\right\|_{L^{p / 2, q / 2}} \approx\|u\|_{L^{p, q}}^{2}$ and the triangle inequality in the Lorentz-space $L^{3 / 2,1}$.

We now turn our attention to the operators $\mathcal{A}_{k}$ and the proof of (9.3). We are fortunate as our inequality involves the space $L^{2}$ at least on the function side and one can reduce matters to the estimation of an oscillatory integral operator

$$
T_{\lambda} f(x)=\int e^{i \lambda \Phi(x, y)} \chi(x, y) f(y) d y
$$

mapping $L^{2}$ to $L^{3,2}$ with norm $O\left(\lambda^{-d / 3}\right)$. Here $\lambda \approx 2^{k}$ and the phase is given by

$$
\Phi(x, y)=y_{2}\left(x_{2}+\frac{\left(x_{1}-y_{1}\right)^{2}}{2}\right)+y_{3}\left(x_{3}+\frac{\left(x_{1}-y_{1}\right)^{3}}{6}\right),
$$

where $\left|y_{3}\right| \geq c>0$ in the support of $\chi$. The reduction to the oscillatory integral operator involves Plancherel's theorem (with respect to the $\left(y_{2}, y_{3}\right)$ variables), a rescaling by $2^{k}$, and renaming $(\sigma, \tau)$ to $\left(y_{2}, y_{3}\right)$.

Now define

$$
T_{\lambda, l} f(x)=\int e^{i \lambda \Phi(x, y)} \chi(x, y) \chi\left(2^{l} \operatorname{det} \Phi_{x y}\right) f(y) d y
$$

where the cutoff function localizes to the set where $\left|\operatorname{det} \Phi_{x y}\right| \approx 2^{-l}$. Observe that

$$
-\operatorname{det} \Phi_{x y}=y_{2}+y_{3}\left(x_{1}-y_{1}\right) \text {. }
$$

Define $\widetilde{T}_{\lambda}$ by a similar cutoff to the region where $\left|\operatorname{det} \Phi_{x y}\right| \leq \lambda^{-1 / 3}$ and choose the cutoff functions so that $T_{\lambda}=\sum_{2^{l}<\lambda^{1 / 3}} T_{\lambda, l}+\widetilde{T}_{\lambda}$.

We have the usual $L^{2}$ bounds $\left\|T_{\lambda, l}\right\|_{L^{2} \rightarrow L^{2}} \lesssim 2^{l / 2} \lambda^{-3 / 2}$ for $2^{l}<\lambda^{1 / 3}$ and $\left\|\widetilde{T}_{\lambda}\right\|_{L^{2} \rightarrow L^{2}} \lesssim$ $\lambda^{-4 / 3}$. Now it remains to show that for $r<4, s=3 r^{\prime}$

$$
\begin{aligned}
\left\|T_{\lambda, l}\right\|_{L^{r} \rightarrow L^{s}} & \lesssim 2^{-l / r^{\prime}} \lambda^{-3 / r}, \quad 2^{l}<\lambda^{1 / 3} \\
\left\|\widetilde{T}_{\lambda}\right\|_{L^{2} \rightarrow L^{2}} & \lesssim \lambda^{-3 / r-1 / 3 r^{\prime}}
\end{aligned}
$$

This implies that $T_{\lambda}$ maps $L^{p, 1}$ to $L^{q, \infty}$ for $1 \leq p<5 / 2, q=3 p^{\prime} / 2$. By another real interpolation we deduce that $T_{\lambda}$ maps in fact $L^{p, a}$ to $L^{q, a}$ for any $a>0$, and choosing $a=p$ and $p=2$ yields the $L^{2} \rightarrow L^{3,2}$ inequality for $T_{\lambda}$.

The proofs of (9.6), (9.7) follow the ideas in Theorem 1.2, however as in $\S 4$ we may directly reduce matters to B. Barceló's restriction theorem for cones ([1]). In fact let for $w \in \mathbb{R}^{3}, v \in \mathbb{R}^{2}, v_{2} \approx 1$

$$
\Psi(w, v)=v_{2}\left(-w_{1} v_{1}^{2}+w_{2} v_{1}+w_{3}\right)
$$


and define $S_{\lambda} f(w)=\int e^{i \lambda \Psi(w, v)} \chi(w, v) f(v) d v$. Then

$$
\left\|S_{\lambda} f\right\|_{s} \lesssim \lambda^{-3 / s}\|f\|_{r}, \quad s=3 r^{\prime}, r<4
$$

Indeed from the restriction theorem we get (9.8) for cutoff's of the product form $\chi(w, v)=$ $a(w) b(v)$, and by using a Fourier series expansion of $\chi$ we can reduce to this case.

In order to prove (9.6) we split $f=\sum_{\kappa \in \mathbb{Z}} f_{\kappa}$ where the function $f_{\kappa}$ is supported in $\left\{y:\left|-y_{1}+y_{2} / y_{3}-\kappa 2^{-l}\right| \leq 2^{-l}\right\}$, and then we observe that $T_{\lambda, l} f_{\kappa}$ is supported where $\left|x_{1}-\kappa 2^{-l}\right| \leq C 2^{-l}$. It thus suffices to show (9.6) for $f$ replaced with $f_{\kappa}$. Fix $\kappa$ and let $\alpha_{\kappa}=\kappa 2^{-l}$. We change variables in $y$ by setting $y_{2}=-y_{3}\left(\alpha_{\kappa}-y_{1}+\sigma\right)$ (where $|\sigma| \lesssim 2^{-l}$ ) and then set $v_{1}=y_{1}, v_{2}=y_{3}$. After a short computation we obtain

$$
\Phi\left(x ; y_{1},-y_{3}\left(\alpha_{\kappa}-y_{1}+\sigma\right), y_{3}\right)-y_{1}^{3} y_{3} / 3=\Psi\left(h^{\sigma}(x), y_{1}, y_{3}\right) \equiv \Psi\left(h^{\sigma}(x), v\right)
$$

with

$$
h^{\sigma}(x)=\left(x_{1}+\sigma-\alpha_{\kappa}, x_{2}-\left(\sigma-\alpha_{\kappa}\right) x_{1}-x_{1}^{2} / 2, x_{3}+\left(\sigma-\alpha_{\kappa}\right)\left(x_{2}+x_{1}^{2} / 2\right)+x_{1}^{3} / 6\right) ;
$$

clearly as a nonlinear shear $h^{\sigma}$ defines a global diffeomorphism.

Now

$$
T_{\lambda, l} f_{\kappa}(x)=\int_{|\sigma| \lesssim 2^{-l}} \int e^{i \lambda \Psi\left(h^{\sigma}(x), v\right)} \chi^{\sigma}(x, v) f_{\kappa, \sigma}(v) d v
$$

with $\chi^{\sigma}(x, v) f_{\kappa, \sigma}(v)=\chi\left(x, v_{1},-v_{2}\left(\alpha_{\kappa}-v_{1}+\sigma\right), v_{2}\right) f_{\kappa}\left(v_{1},-v_{2}\left(\alpha_{\kappa}-v_{1}+\sigma\right), v_{2}\right) e^{i \lambda v_{1}^{3} v_{2} / 3}$. Thus from (9.8) we get

$$
\left\|T_{\lambda, l} f_{\kappa}\right\|_{L^{s}\left(\mathbb{R}^{3}\right)} \lesssim \int_{|\sigma| \lesssim 2^{-l}}\left\|f_{\kappa, \sigma}\right\|_{L^{r}\left(\mathbb{R}^{2}\right)} d \sigma \lesssim 2^{-l / r^{\prime}}\left(\int\left\|f_{\kappa, \sigma}\right\|_{L^{r}\left(\mathbb{R}^{2}\right)}^{r} d \sigma\right)^{1 / r} \lesssim 2^{-l / r^{\prime}}\left\|f_{\kappa}\right\|_{L^{r}\left(\mathbb{R}^{3}\right)}
$$

which implies (9.6). Inequality (9.7) is proved in a similar way.

\section{APPENDIX II:}

ON BILINEAR VERSIONS OF AN ADJOINT RESTRICTION THEOREM FOR THE CIRCLE

Let $d \sigma$ denote arclength measure on the circle and for $f \in L^{2}\left(\mathbb{S}^{1}\right)$ consider the family of (restricted) extension operators given by $\mathcal{E}_{\lambda} f(\theta)=\widehat{f d \sigma}(\lambda \theta)$, where $\theta \in \mathbb{S}^{1}$. In 2] Barceló, Carbery and one of the current authors proved the sharp bilinear inequality

$$
\int\left|\mathcal{E}_{\lambda} f(\theta) \mathcal{E}_{\lambda} g(\theta)\right| d \sigma(\theta) \leq C \lambda^{-5 / 6}\|f\|_{L^{2}\left(\mathbb{S}^{1}\right)}\|g\|_{L^{2}\left(\mathbb{S}^{1}\right)}
$$

valid under the separation conditions

$$
\operatorname{supp}(f) \cap \operatorname{supp}(g)=\emptyset \text { and } \operatorname{supp}(\widetilde{f}) \cap \operatorname{supp}(g)=\emptyset ;
$$

here $\widetilde{f}(\theta):=f(-\theta)$. The initially complicated proof of this inequality in [2] has since been simplified in [3. Here we generalize and simplify further this result by interpreting the separation condition (10.1) as a condition on the associated canonical relation, and deduce the bilinear estimates directly from known linear estimates of the type (4.3), (4.4) (proved already in 32 for $d=1$ ). Several multilinear extensions of this argument are possible (in the spirit of [3] ) but we shall not pursue this here.

Consider the oscillatory integral operator defined on functions in $L^{2}(\mathbb{R})$ by

$$
T_{\lambda} f(x)=\int e^{i \lambda \phi(x, y)} \chi(x, y) f(y) d y
$$


where $\chi$ is smooth and compactly supported. We assume that the canonical relation $\left\{\left(x, \phi_{x}, y,-\phi_{y}\right)\right\}$ is a folding canonical relation, i.e. (1.7) and (1.8) hold. We may clearly assume that $\phi_{x y}$ is small on the support of the amplitude.

Proposition 10.1. Suppose that $\chi$ is supported on a set of diameter at most $\delta_{0}$ and suppose that $\phi_{x y y} \neq 0$ and $\phi_{x x y} \neq 0$ on the support of $\chi$.

Let $0<\delta<\delta_{0}$ and suppose that

$$
\operatorname{dist}(\operatorname{supp} f, \operatorname{supp} g) \geq \delta>0 \text {. }
$$

Then, if $\delta_{0}$ is sufficiently small then, for large $\lambda>0$,

$$
\left\|T_{\lambda} f T_{\lambda} g\right\|_{1} \leq C_{\delta} \lambda^{-5 / 6}\|f\|_{2}\|g\|_{2}
$$

(ii) Moreover

$$
\left\|T_{\lambda} f T_{\lambda} g\right\|_{6 / 5} \leq C_{\delta} \lambda^{-5 / 6}\left[\|f\|_{2}\|g\|_{3}+\|f\|_{3}\|g\|_{2}\right]
$$

Remark: The inequality (10.1) (valid assuming (10.2) ) can be deduced by applying the proposition with the phase $\phi(x, y)=\cos (x-y)$.

Proof of Proposition 10.1. We may assume that $\lambda \gg \delta^{-1}$. A better inequality follows immediately by the standard $L^{2}$ estimates if we assume that $\phi_{x y} \neq 0$; thus we shall assume that $\phi_{x y}$ vanishes somewhere and after a straightforward reduction (using suitable localizations) we may assume that on the support of the relevant cutoff function $\phi_{x y}=0 \Longleftrightarrow x=h(y)$ where $h$ is invertible and $\left|h^{\prime}\right|$ is bounded above and below (actually, as in 32] one can reduce to $h(y)=y$, by a change of variable in $y$ ).

Let $\chi_{0} \in C_{0}^{\infty}(\mathbb{R})$ be supported in $(-1,1)$ and equal to one in $(-1 / 2,1 / 2)$, and let $\chi_{1}(t)=$ $\chi(2 t)-\chi(t), \chi_{l}(t)=\chi_{1}\left(2^{l} t\right)$. Consider the operators given by $T_{\lambda, l}$ and $\widetilde{T}_{\lambda}$ defined in and following (4.2). Notice that the kernel $K_{\lambda, l}(x, y)$ is supported where $|x-h(y)| \approx 2^{-l}$, and and similarly the kernel for $S_{\lambda}$ is supported where $|x-h(y)| \lesssim \lambda^{-1 / 3}$.

Now write

$$
T_{\lambda} f(x) T_{\lambda} g(x)=\left(\widetilde{T}_{\lambda} f(x)+\sum_{2^{l} \leq \lambda^{1 / 3}} T_{\lambda, l} f(x)\right)\left(\widetilde{T}_{\lambda} g(x)+\sum_{2^{m} \leq \lambda^{1 / 3}} T_{\lambda, m} g(x)\right) .
$$

We use the separation assumption on the supports of $f$ and $g$. If $y \in \operatorname{supp} f$ and $z \in \operatorname{supp} g$ then the conditions $|x-h(y)| \approx 2^{-l}$, and $|x-h(z)| \approx 2^{-m}$, can hold simultaneously only if either $l \leq \ell_{0}$, or $m \leq \ell_{0}$, for some fixed $\ell_{0}=\ell_{0}(\delta)$. Thus

$$
T_{\lambda, l} f(x) T_{\lambda, m} g(x)=0 \quad \text { if } l \geq \ell_{0}, \text { and } m \geq \ell_{0}
$$

Similarly $\widetilde{T}_{\lambda} f(x) \widetilde{T}_{\lambda} g(x)=0$, and $\widetilde{T}_{\lambda} f(x) T_{\lambda, l} g(x)=0, l \leq \ell_{0}$. Therefore,

$$
T_{\lambda} f T_{\lambda} g=I_{\lambda}(f, g)+I I_{\lambda}(f, g)+I I I_{\lambda}(f, g)
$$


where

$$
\begin{aligned}
I_{\lambda}(f, g) & =\left(\widetilde{T}_{\lambda} f+\sum_{2^{\ell_{0}<2^{l} \leq \lambda^{1 / 3}}} T_{\lambda, l} f\right)\left(\sum_{m \leq \ell_{0}(\delta)} T_{\lambda, m} g\right), \\
I I_{\lambda}(f, g) & =\left(\sum_{l \leq \ell_{0}(\delta)} T_{\lambda, l} f\right)\left(\widetilde{T}_{\lambda} g+\sum_{2^{\ell_{0}<2^{m} \leq \lambda^{1 / 3}}} T_{\lambda, l} g\right), \\
I I I_{\lambda}(f, g) & =\left(\sum_{l \leq \ell_{0}(\delta)} T_{\lambda, l} f\right)\left(\sum_{m \leq \ell_{0}(\delta)} T_{\lambda, l} g\right) .
\end{aligned}
$$

We now use the Cauchy-Schwarz inequality and apply the standard $L^{2}$ bounds (4.3), (4.4) (with $d=1$ ). We obtain

$$
\begin{aligned}
\left\|I_{\lambda}(f, g)\right\|_{1} & \leq\left(\left\|\widetilde{T}_{\lambda} f\right\|_{2}+\sum_{2^{\ell_{0}<2^{l} \leq \lambda^{1 / 3}}}\left\|T_{\lambda, l} f\right\|_{2}\right) \sum_{m \leq \ell_{0}(\delta)}\left\|T_{\lambda, m} g\right\|_{2} \\
& \leq C(\delta) \lambda^{-1 / 2}\left(\lambda^{-1 / 3}+\sum_{2^{\ell_{0}<2^{l} \leq \lambda^{1 / 3}}} 2^{l / 2} \lambda^{-1 / 2}\right)\|f\|_{2}\|g\|_{2} \leq C_{1}(\delta) \lambda^{-5 / 6}\|f\|_{2}\|g\|_{2} .
\end{aligned}
$$

Similarly one proves the inequality $\left\|I I_{\lambda}(f, g)\right\|_{1} \leq C_{2}(\delta) \lambda^{-5 / 6}\|f\|_{2}\|g\|_{2}$, and also the bound $\left\|I I I_{\lambda}(f, g)\right\|_{1} \leq C_{3}(\delta) \lambda^{-1}\|f\|_{2}\|g\|_{2}$. This shows (10.4).

For (10.5) we use the endpoint $L^{3} \rightarrow L^{3}$ inequality in [22] which says that

$$
\left\|\sum_{2^{\ell_{0}<2^{l} \leq \lambda^{1 / 3}}} T_{\lambda, l} f\right\|_{3} \leq C_{\delta} \lambda^{-1 / 3}\|f\|_{3}
$$

and also $\left\|S_{\lambda}\right\|_{L^{3} \rightarrow L^{3}}=O\left(\lambda^{-1 / 3}\right)$. Since $L^{2} \cdot L^{3} \subset L^{6 / 5}$ we apply Hölder's inequality to obtain

$$
\begin{aligned}
\left\|I_{\lambda}(f, g)\right\|_{6 / 5} & \leq C_{\delta} \lambda^{-5 / 6}\|f\|_{3}\|g\|_{2}, \\
\left\|I I_{\lambda}(f, g)\right\|_{6 / 5} & \leq C_{\delta} \lambda^{-5 / 6}\|f\|_{2}\|g\|_{3}, \\
\left\|I I I_{\lambda}(f, g)\right\|_{6 / 5} & \leq C_{\delta} \lambda^{-5 / 6}\|f\|_{2}\|g\|_{2},
\end{aligned}
$$

and thus (10.5).

\section{REFERENCES}

[1] B. Barceló, On the restriction of the Fourier transform to a conical surface, Trans. Amer. Math. Soc. 292, No. 1 (1985), 321-333.

[2] J. A. Barceló, J. M. Bennett and A. Carbery, A bilinear extension inequality in two dimensions, J. Funct. Anal. 201 (2003), no. 1, 57-77.

[3] _ A multilinear extension inequality in $\mathbb{R}^{n}$, Bull. London Math. Soc. 36 (2004), no. 3, 407-412.

[4] J. A. Barceló, A. Ruiz and L. Vega, Weighted estimates for the Helmholtz equation and consequences, J. Funct. Anal. 150 (1997), no. 2, 356-382.

[5] W. Beckner, A. Carbery, S. Semmes, and F. Soria, A note on restriction of the Fourier transform to spheres, Bull. London Math. Soc. 21 (1989), no. 4, 394-398.

[6] J. M. Bennett, A. Carbery, F. Soria and A. Vargas, A Stein conjecture for the circle, to appear in Math. Annalen.

[7] J. Bourgain, Estimations de certaines fonctions maximales, C. R. Acad. Sci. Paris Sér. I Math. 301 (1985), no. 10, 499-502.

[8] Besicovitch type maximal operators and applications to Fourier analysis, Geom. Funct. Anal. 1 (1991), 147-187.

[9] L $^{p}$-estimates for oscillatory integrals in several variables, Geom. Funct. Anal. 1 (1991), no. 4, $321-374$. 
[10] A. Carbery and A. Seeger, Weighted inequalities for Bochner-Riesz means in the plane, Quart. Jour. Math. 51 (2000), no. 2, 155-167.

[11] A. Carbery, A. Seeger, S. Wainger and J. Wright, Classes of singular integral operators along variable lines, J. Geom. Anal. 9 (1999), no. 4, 583-605.

[12] A. Carbery and F. Soria, Pointwise Fourier inversion and localisation in $\mathbb{R}^{n}$, Journal of Fourier Analysis and Applications 3, special issue, 847-858 (1997).

[13] _ Sets of divergence for the localisation principle for Fourier integrals, C. R. Acad. Sci. Paris. Sér. I Math. 325 (1997), no. 12, 1283-1286.

[14] L. Carleson and P. Sjölin, Oscillatory integrals and a multiplier problem for the disc, Studia Math. 44 (1972), 287-299.

[15] A. Comech, Sobolev estimates for the Radon transform of Melrose and Taylor. Comm. Pure Appl. Math. 51 (1998), no. 5, 537-550.

[16] Optimal estimates for Fourier integral operators with one-sided folds, Comm. Part. Diff. Eqs. 24 (1999), 1263-1281.

[17] S. Cuccagna, $L^{2}$ estimates for averaging operators along curves with two-sided $k$-fold singularities, Duke Math. J. 89 (1997), no. 2, 203-216.

[18] M. B. Erdoğan, A bilinear Fourier extension theorem and applications to the distance set problem, Int. Math. Res. Not. 23 (2005), 1411-1425.

[19] - On Falconer's distance conjecture, Rev. Iberoamericana, to appear.

[20] C. Fefferman, Inequalities for strongly singular convolution operators, Acta Math. 124 (1970), 9-36.

[21] A. Greenleaf and A. Seeger, Fourier integral operators with fold singularities, J. Reine Angew. Math. 455 (1994), 35-56.

[22] _ On oscillatory integral operators with folding canonical relations, Studia Math. 132 (1999), no. 2, 125-139.

[23] - Oscillatory and Fourier integral operators with degenerate canonical relations, Proceedings of the 6th International Conference on Harmonic Analysis and Partial Differential Equations (El Escorial, 2000). Publ. Mat. 2002, Vol. Extra, 93-141.

[24] L. Hörmander, Oscillatory integrals and multipliers on $F L^{p}$, Ark. Mat. 11 (1973), 1-11.

[25] U. Keich, On $L^{p}$ bounds for Kakeya maximal functions and the Minkowski dimension in $\mathbb{R}^{2}$, Bull. London Math. Soc. 31 (1999), no. 2, 213-221.

[26] P. Mattila, Hausdorff dimension, projections, and the Fourier transform, Publ. Mat. 48 (2004), 3-48.

[27] R. Melrose and M. Taylor, Near peak scattering and the corrected Kirchhoff approximation for a convex obstacle, Adv. in Math. 55 (1985), no. 3, 242-315.

[28] G. Mockenhaupt, A. Seeger and C. D. Sogge, Local smoothing of Fourier integral operators and Carleson-Sjölin estimates, J. Amer. Math. Soc. 6 (1993), no. 1, 65-130.

[29] D. Müller and A. Seeger, Regularity properties of wave propagation on conic manifolds and applications to spectral multipliers, Advances in Mathematics, 161 (2001), 41-130.

[30] D. Oberlin, Convolution estimates for some measures on curves, Proc. Amer. Math. Soc. 99 (1987), no. 1, 56-60.

[31] Y. Pan and C. D. Sogge, Oscillatory integrals associated to folding canonical relations, Colloq. Math. 60/61 (1990), 413-419.

[32] D. H. Phong and E. M. Stein, Radon transforms and torsion, Internat. Math. Res. Notices 1991, no. 4, 49-60.

[33] A. Ruiz and L. Vega, Unique continuation for Schrödinger operators with potential in Morrey spaces, Publ. Mat., 35 (1991), 291-298, and Publ. Mat. 39 (1995), 405-411.

[34] A. Seeger, Endpoint inequalities for Bochner-Riesz multipliers in the plane, Pacific J. Math. 174 (1996), 543-553.

[35] P. Sjölin and F. Soria, Estimates of spherical averages of Fourier transforms and dimensions of sets, Proc. Roy. Soc. Edinburgh Sect. A 133 (2003), no. 4, 943-950.

[36] E. M. Stein, Oscillatory integrals in Fourier analysis, Beijing Lectures in Harmonic Analysis, Princeton Univ. Press, Princeton, N.J. (1986), 307-356.

[37] T. Tao, Sharpness of the Carleson-Sjölin theorem, expository note, 2001.

[38] _ A sharp bilinear restriction estimate for paraboloids, Geom. Funct. Anal. 13 (2003), no. 6, 1359-1384.

[39] P. Tomas, Restriction theorems for the Fourier transform, Proc. Symp. Pure Math., (1979), 111-114. 
[40] T. H. Wolff, Recent work connected with the Kakeya problem, Prospects in mathematics (Princeton, NJ, 1996), 129-162, Amer. Math. Soc., Providence, RI, 1999.

[41] _ Decay of circular means of Fourier transforms of measures, Internat. Math. Res. Notices 10 (1999), 547-567; Addendum in J. Anal. Math. 88 (2002), 35-39.

Jonathan Bennett, School of Mathematics, University of Birmingham, The Watson Building, Edgbaston, Birmingham, B15 2TT, United Kingdom

E-mail address: J.Bennett@bham.ac.uk

Andreas Seeger, Department of Mathematics, University of Wisconsin, Madison, Wisconsin 53706-1388, USA

E-mail address: seeger@math.wisc.edu 